\title{
Seasonal Variations of the Urban Thermal Environment Effect in a Tropical Coastal City
}

\author{
Yao Yuan, ${ }^{1,2,3}$ Chen Xi, ${ }^{1}$ Qian Jing, ${ }^{2}$ and Ndayisaba Felix ${ }^{3,4,5}$ \\ ${ }^{1}$ State Key Laboratory of Desert and Oasis Ecology, Xinjiang Institute of Ecology and Geography, Chinese Academy of Sciences, \\ Urumqi 830011, China \\ ${ }^{2}$ Shenzhen Institute of Advanced Technology, Chinese Academy of Sciences, Shenzhen 518055, China \\ ${ }^{3}$ University of Chinese Academy of Sciences, Beijing 100049, China \\ ${ }^{4}$ Faculty of Environmental Sciences, University of Lay Adventists of Kigali (UNILAK), P.O. Box 6392, Kigali, Rwanda \\ ${ }^{5}$ Institute of Remote Sensing and Digital Earth, Chinese Academy of Sciences, Beijing 100094, China \\ Correspondence should be addressed to Qian Jing; jing.qian@siat.ac.cn
}

Received 1 June 2017; Revised 4 September 2017; Accepted 11 October 2017; Published 12 November 2017

Academic Editor: Jorge E. Gonzalez

Copyright ( 2017 Yao Yuan et al. This is an open access article distributed under the Creative Commons Attribution License, which permits unrestricted use, distribution, and reproduction in any medium, provided the original work is properly cited.

\begin{abstract}
Shenzhen city was selected to analyze the Surface Urban Heat Island (SUHI) variations based on land surface temperature (LST) in four different seasons of 2015. UHI intensity (UHII) as an indicator of SUHI was established and the method of density segmentation was utilized to classify the SUHI after LSTs were normalized. The gravity center model of UHII and Moran's $I$ (a spatial autocorrelation index) were used to analyze the spatiotemporal variations of SUHI. Results indicated that LST was higher in the west than in the east of the city. The values of UHII were higher in spring and summer and lower in autumn and winter. Five profiles were drawn to analyze the distribution of UHII in different seasons, and it was found that the No. 1 path profiles, corresponding to the western urban development axis, had higher UHII than other path profiles. The center of UHII gravity shifts converged in the Longhua, Baoan, and Nanshan Districts throughout the four seasons and Moran's $I$ values were higher in summer and spring. From the UHII's spatial distribution pattern analysis, a spatially discontinuous pattern was observed in four seasons; there was a compact pattern of high temperature zones.
\end{abstract}

\section{Introduction}

Urban heat island (UHI) effect refers to the phenomenon by which urban areas' temperatures become higher than those of the surrounding rural areas, usually as a result of rapid urbanization [1-3]. Based on the UHI concept, urban thermal environment is a concept that was put forward by experts and scholars in meteorology and environmental research field in recent years $[4,5]$, referring to the heat-related physical environment that can affect the urban atmosphere, energy consumption, body's sense of well-being, health status, and human survival and development [6]. At present, the deterioration of urban thermal environment has become one of the most significant features of global urban climate change and has great negative impacts on urban air quality improvement, $\mathrm{CO}_{2}$, and haze control, as well as plant health and growth [7, 8]. The evolution of urban thermal environment has a close relationship with human society and economic activities. The acceleration of the urbanization process and the expansion of asphalt, metal, cement, and other impervious surfaces at the expense of natural surfaces have, in conjunction with population growth, resulted in urban climate change, which is even more dramatic than global change $[9,10]$.

In general, UHI can be used to reflect and embody the urban thermal environment [11]. Oke (1995) and Yow (2007) demonstrated that UHI could be divided into three types: Canopy Layer Heat Island (CLHI), Boundary Layer Heat Island (BLHI), and Surface Urban Heat Island (SUHI) $[12,13]$ whereby the first two types refer to atmospheric urban heat island. The Urban Canopy Layer (UCL) roughly refers to the layer from the ground surface to the urban elements' height (mainly tree or building) while the Urban Boundary Layer (UBL) refers to the layer situated directly above the UCL and in the layer of the atmosphere $[14,15]$. 
These two distinct layers' heat island studies primarily utilize air temperature data or meteorological datasets with ground observation and numerical simulation as the main methodological approaches involved [16, 17]. SUHI differs from atmospheric urban heat island and is usually measured by surface temperature (LST) data retrieved from thermal infrared airborne and satellite sensors. The SUHI is substantially affected by urbanization and has been increasingly used in recent studies because it is closely related to human health and directly linked to land surface features $[18,19]$.

Remote sensing technology has the advantages of short acquisition period, wide coverage, low user cost, and rapid and accurate monitoring of urban surface temperatures [20, 21]. It has presently become the main technical means and a powerful research tool for experts and scholars to analyze the trends and dynamics of UHI, owing to the fact that it can give a spatially continuous view of the SUHI and effectively depict the patterns of the thermal environment over large urban areas compared to that from air temperature measured at standard meteorological stations or groundbased air temperature measurements in cities [22-24].

Early efforts started in 1972 when, for the first time, Rao used thermal infrared remote sensing technology to study the surface temperature distribution model and SUHI in coastal cities of the Atlantic Ocean in the United States [25]. Since then, polar orbiting satellites such as AVHRR, MODIS, Landsat TM/ETM, and geostationary orbiting satellites (e.g., GOES, MSG) have been the most commonly used remote sensing data for SUHI research, with advantages of measuring urban thermal environments and offering spatially explicit coverage at multiple scales, ranging from cities to the whole world [26, 27]. Nevertheless, the design of these satellite sensors has been regarded as a bottleneck limiting their application for SUHI study, because remote sensing imagery has a high temporal resolution but a relatively low spatial resolution [28]. For instance, SEVIRI onboard MSG and the GOES satellites can collect data every 5 and 30 minutes, respectively, which is fairly adequate to monitor the diurnal development of LST [29] but are characterized by very low spatial resolutions of about $3000 \mathrm{~m}$. In order to describe the UHI characteristics, downscaling methods have often been employed to enhance the spatial resolution, but the choice of appropriate method still remains a great challenge [30]. Likewise, AVHRR and MODIS images have relatively higher temporal resolutions but their low spatial resolution constrains their use. For instance, AVHRR has an overpass with two images at the equator every day, but their low spatial resolution $(1.1 \mathrm{~km})$ is too coarse to study variations in SUHI with precision [31]. Similarly, MODIS acquires data at a high temporal resolution, but it is still problematic to accurately describe the spatial changes in SUHI due to the relatively low spatial resolution $(1 \mathrm{~km})[32,33]$. Numerous SUHI studies based on Landsat TM/ETM+ data have been conducted but the spatial resolution of Landsat TM/ETM+ data is still relatively high $(120 \mathrm{~m} / 60 \mathrm{~m})$ while its temporal resolution is low (16 days), which makes it extremely difficult to obtain the desired image for analysis and monitoring SUHI dynamics in real time [34-36]. Therefore, it is imperative to seek an alternative remote sensing dataset with a rather satisfactory spatiotemporal resolution. HJ-1B is a satellite successfully launched by China on September 6, 2008, whose main function lies in environmental disaster monitoring and forecasting. The spatial resolution of the thermal infrared band IRS4 is $300 \mathrm{~m}$, which can meet the requirements of the SUHI monitoring on the spatial scale. The revisit time of the satellite is 4 days which is enough for it to perform global coverage and the possibility of data acquisition at a much higher frequency than that of Landsat instruments. Over the recent years, Ouyang et al. [37] used HJ-1B remote sensing imagery to retrieve LST over the Heihe river basin in China, while Wu et al. [28] used HJ-1B thermal infrared bands to assess the effects of land use spatial structure on UHI in Wuhan, China. Notwithstanding these studies however, none focused on the seasonal variations of SUHI, especially in areas with remarkably rapid urbanization processes such as Shenzhen, one of the most rapidly urbanizing cities in China.

The objective of this study is twofold. Firstly, it examines the spatial characteristics of the SUHI change in Shenzhen, as quantified by LST and urban heat island intensity (UHII), which are two important indicators to evaluate the severity of SUHI in different seasons. Secondly, it analyzes and discusses the seasonal variations of SUHI in Shenzhen during 2015. Remote sensing images were used to retrieve the seasonal LST, which was further classified into seven levels to show the SUHI intensity. Spatial analysis including Moran's $I$ and gravity center model were conducted to derive the spatial patterns and dynamics of seasonal SUHI change on local scales. Based on the results and discussion, this paper may provide useful information for urban planners to create strategies to mitigate SUHI effects in Shenzhen.

\section{Materials and Methods}

2.1. Study Area. The city of Shenzhen is located between $113^{\circ} 46^{\prime} \mathrm{E} \sim 114^{\circ} 37^{\prime} \mathrm{E}$ and $22^{\circ} 27^{\prime} \mathrm{N} \sim 22^{\circ} 52^{\prime} \mathrm{N}$ in the southern part of Guangdong Province of China, where it shares borders with the Hong Kong Special Administrative Region (SAR) to the south; Dongguan, Guangzhou, and Huizhou cities to the north; Pearl River Estuary to the west; and Daya Bay and Dapeng Bay to the east (Figure 1).

Shenzhen has a tropical maritime climate consisting of long summers and short winters. The annual average temperature is $23.0^{\circ} \mathrm{C}$, the average low temperature is $15.4^{\circ} \mathrm{C}$ in January, and the average maximum temperature is $28.9^{\circ} \mathrm{C}$ in July [38]. The mean annual total precipitation is $1933.3 \mathrm{~mm}$, with the rainy season extending from April to September.

Shenzhen city, as a prototype of rapid urbanization since China's reform and opening up policy in 1978, is one of the largest cities in China with the fastest development. Shenzhen has emerged from a border town into an international megacity comprising ten administrative districts. In 2015, the total area of the city was $1996 \mathrm{sq}$. $\mathrm{km}$ while the residing population was estimated at 10.77 million, and the population density and population growth were 604 people $/ \mathrm{km}^{2}$ and $1.4 \%$ in 2015, respectively. The gross domestic product (GDP) was 1750.299 billion yuan. Therefore, Shenzhen can be considered as an ideal area for studying the spatial temporal variability of UHI effect within the context of rapid urbanization. 


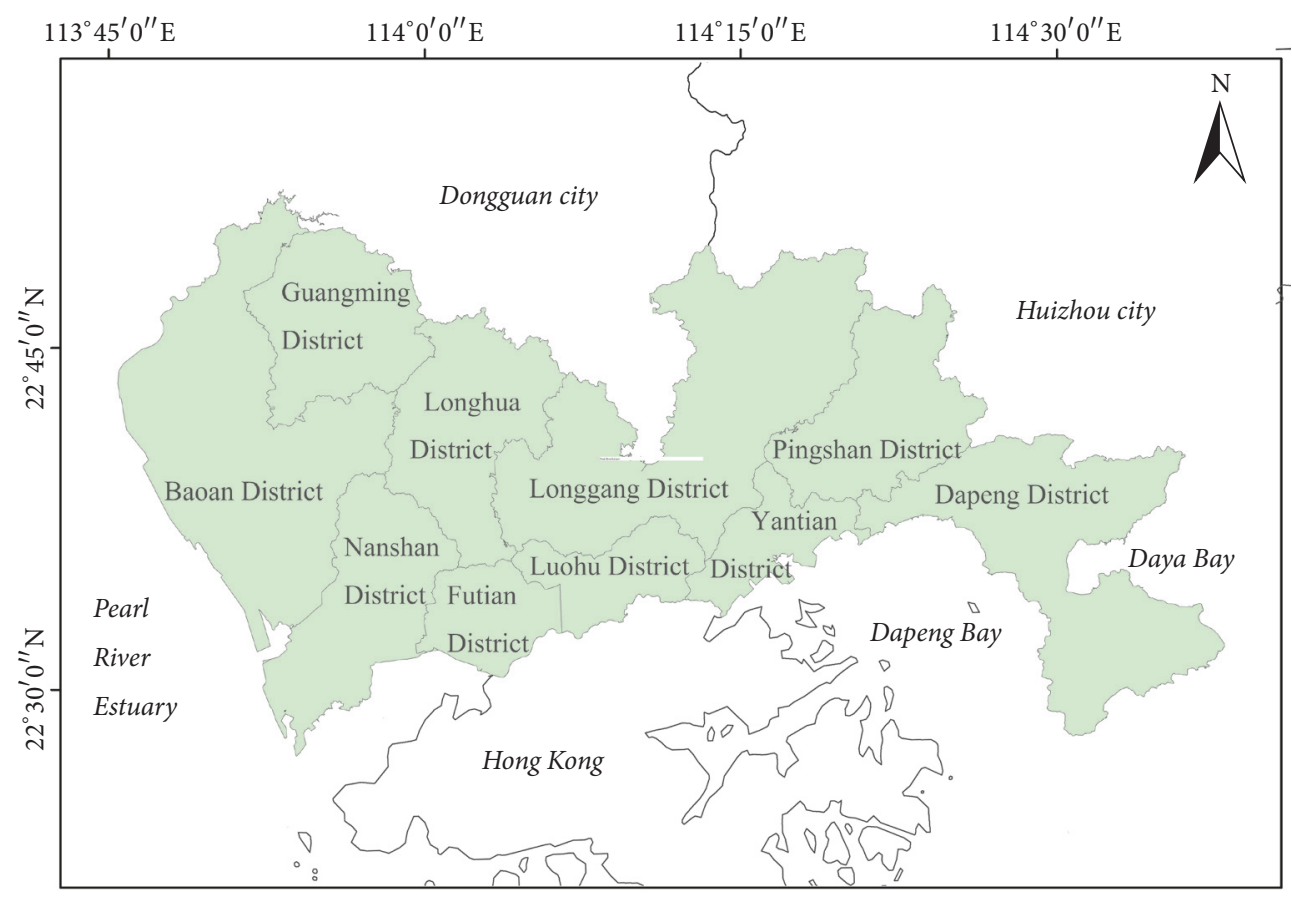

FIGURE 1: Location of the study area.

TABLE 1: Main parameters of HJ-1B satellite sensor.

\begin{tabular}{lccc}
\hline Parameters & CCD1 and CCD2 & \multicolumn{2}{c}{ IRS } \\
\hline Band & $1-4$ & $5-7$ & 8 \\
Spectral range $(\mu \mathrm{m})$ & $0.43-0.90$ & $0.75-3.90$ & $10.5-12.5$ \\
Spatial resolution $(\mathrm{m})$ & 30 & 150 & 300 \\
Amplitude width $(\mathrm{km})$ & 360 & \multicolumn{2}{c}{720} \\
Revisit cycle (days) & 2 & \multicolumn{2}{c}{4} \\
\hline
\end{tabular}

2.2. Data Source and Preprocessing. HJ-1B is a small optical satellite that was launched in September 6, 2008. It is mainly used for monitoring the environment and disasters, owing to the Chinese government's will to enhance disaster reduction and risk control capabilities and improve environmental protection [39]. HJ-1B satellite has one infrared sensor (IRS) and two charge-coupled device (CCD) sensors. The IRS can provide global coverage every 4 days, while the CCD sensor can cover the globe in 2 days [40]. The CCD sensors have four bands: band 1 is blue, and band 2 to band 4 are green, red, and infrared bands, respectively. The IRS has four bands including band 5 (near infrared band, NIR), band 6 (shortwave infrared band, SWIR), band 7 (middle-wave infrared band, MWIR), and band 8 (longwave band, LWIR). The main parameters of the $\mathrm{HJ}-1 \mathrm{~B}$ satellite include the $30 \mathrm{~m}$ spatial resolution CCD data and $300 \mathrm{~m}$ spatial resolution thermal infrared data [41]. The detailed sensor parameters are shown in Table 1.

In 2015, using the 5-Day Running Mean Temperature method based on the meteorological data (1980-2010) of Shenzhen city, the Meteorological Bureau of Shenzhen Municipality announced the succession of seasons in the order of winter (January 13th to February 6th), spring (February 6th to April 21st), summer (April 21st to November 3rd), and the fall (November 3rd to January 13th) [42]. Since then, many studies have shown that the method used was appropriate in relation to the meteorological characteristics of Shenzhen [43]. As it often occurs in tropical areas, climatic conditions of the tropical coastal cities like Shenzhen are less than ideal for seasonal SUHI study because the images are often occluded by clouds $[44,45]$. Therefore, despite nearly 10 years of HJ-1B imagery only four high quality HJ-1B CCD and IRS images were deemed usable and were selected for use in this study, respective of seasons. These images were acquired in winter, spring, summer, and autumn of 2015, precisely on January 16, April 14, October 18, and December 18, respectively. These images were acquired at approximately 11:05 am local time under steady atmospheric conditions favorable for SUHI studies such as windless weather and sunny conditions. In order to further ensure their suitability for seasonal analysis and prove that the date of the remote sensing images acquisition is not affected by extreme temperature [46], we compared the daily maximum and minimum air temperature derived from the acquired images with the average maximum and minimum air temperature data provided by the Meteorological Bureau of Shenzhen Municipality as illustrated in Table 2. It can be observed that, by comparison, the daily maximum and minimum air temperature data are within the range of the seasonal average maximum and minimum temperatures, which confers some degree of reliability to the remote sensing data used in this study.

The four CCD images and four IRS images were obtained from the China Center for Resources Satellite Data and 


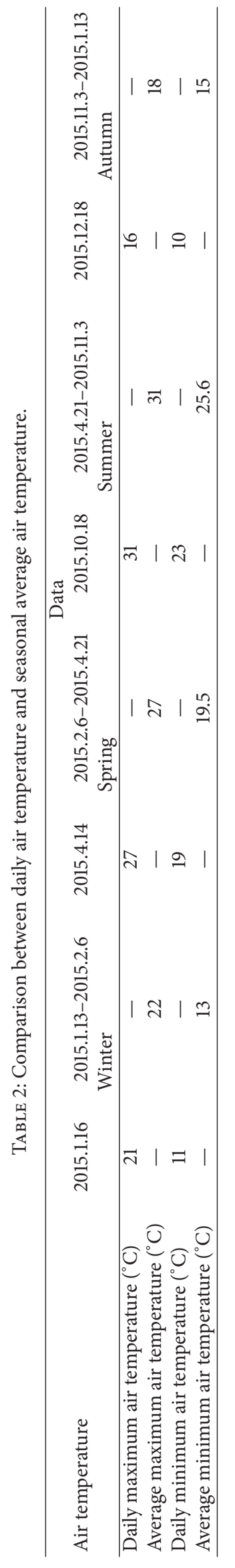


TABLE 3: Absolute radiometric calibration coefficients of HJ-1B CCD and IRS camera.

\begin{tabular}{|c|c|c|c|c|c|c|c|c|}
\hline \multirow{3}{*}{ Band $(\mu \mathrm{m})$} & \multicolumn{8}{|c|}{ Date } \\
\hline & \multicolumn{2}{|c|}{ 2015.1.16 } & \multicolumn{2}{|c|}{ 2015.4.14 } & \multicolumn{2}{|c|}{2015.10 .18} & \multicolumn{2}{|c|}{2015.12 .18} \\
\hline & $g$ & $b$ & $g$ & $b$ & $g$ & $b$ & $g$ & $b$ \\
\hline Band $1(0.48)$ & 0.706 & 3.0089 & 0.8042 & 2.2219 & 0.7060 & 3.0089 & 0.8042 & 2.2219 \\
\hline Band $2(0.565)$ & 0.6960 & 4.4487 & 0.7822 & 4.0683 & 0.6960 & 4.4487 & 0.7822 & 4.0683 \\
\hline Band 3 (0.66) & 1.0082 & 3.2144 & 1.0556 & 5.2537 & 1.0082 & 3.2144 & 1.0556 & 5.2537 \\
\hline Band 4 (0.83) & 1.0068 & 2.5609 & 0.9237 & 6.3497 & 1.0068 & 2.5609 & 0.9237 & 6.3497 \\
\hline IRS4 (11.5) & 56.277 & 12.625 & 56.277 & 12.625 & 56.277 & 12.625 & 56.277 & 12.625 \\
\hline
\end{tabular}

Application, which corrects images for geometrical distortions before delivering a level-2 output product. The preprocessing procedure for the $\mathrm{HJ}-1 \mathrm{~B}$ data includes radiometric calibration, atmospheric correction, and geometric precision correction.

(I) Radiometric Calibration. The radiometric calibration equation used is as follows:

$$
\begin{aligned}
& L_{\mathrm{CCD}}=\frac{\mathrm{DN}}{g}+b, \\
& L_{\mathrm{IRS} 4}=\frac{\mathrm{DN}-b}{g},
\end{aligned}
$$

where $L_{\mathrm{CCD}}$ and $L_{\mathrm{IRS} 4}$ are the radiance $\left(\mathrm{W} \cdot \mathrm{m}^{-2} \cdot \mathrm{sr}^{-1} \cdot \mu \mathrm{m}^{-1}\right)$, $g$ is the gain coefficient, $b$ is the offset, and DN is the image pixel digital values. To convert the $\mathrm{DN}$ values to radiance, we used the HJ-1B CCD and IRS Camera's absolute radiometric calibration coefficients which were obtained from the China Center for Resources Satellite Data and Application (Table 3). The DN values for CCD camera's bands $1-4$ were converted to radiance values using (1), whereas the DN values for IRS's band 4 were converted to radiance values using (2).

(II) Atmospheric Correction. In order to eliminate the influence of atmospheric and light factors on ground reflections, it is necessary to correct the remotely sensed image after calibration. FLAASH is an atmospheric correction module developed by the Spectral Sciences Institute. It directly transfers the radiation transmission calculation method from Modtran5 atmospheric radiation transmission model and accurately retrieves the surface reflectivity from the remote sensing image. FLAASH is widely used in surface temperature research, and its atmospheric correction wavelengths range from $0.4 \mu \mathrm{m}$ to $3 \mu \mathrm{m}$. Hence, ENVI-FLAASH atmospheric correction module was used to calibrate the HJ-1B CCD image after radiometric calibration.

(III) Geometric Correction. Landsat-8 images acquired on October 5 and 15, 2014, were chosen as the reference image, and the $\mathrm{HJ}-1 \mathrm{~B}$ images were corrected using geometric calibration with a correction error of 0.5 pixels or less.

2.3. Retrieval of LST. At present, various LST retrieval methods such as mono-window algorithm [47], split-window algorithm [48], temperature/emissivity separation [49], and single-channel methods [50] have been theoretically developed. Although all of these methods can calculate LST from thermal remote sensing images and provide good results, it has been proven that, by comparison, the mono-window algorithm constitutes a simple and highly effective method for the analysis of SUHI effect [51]. Sobrino et al. (2004) showed that the mono-window algorithm seems to be more applicable than the single-channel method with root mean square deviation of $0.9 \mathrm{~K}$ [52]. Besides, the mono-window algorithm is believed to have a comparative advantage over single-channel algorithms in a sense that it can yield better results in regions with humid atmosphere [53]. Previous researchers have applied the mono-window algorithm for the analysis of SUHI in humid areas like Hong Kong, Casablanca, and Pearl River Delta Region in South China [34, 54, 55]. Thus, the mono-window algorithm has been utilized to retrieve the LST of Shenzhen from HJ-1B data in this study.

The equation for LST retrieval from HJ-1B IRS4 data is as follows:

$$
\begin{aligned}
& T_{S} \\
& =\frac{\left\{a(1-C-D)+[b(1-C-D)+C+D] T_{0}-D T_{a}\right\}}{C}, \\
& C=\varepsilon \tau, D=(1-\tau)[1+(1-\varepsilon) \tau],
\end{aligned}
$$

where $T_{S}$ is land surface temperature in Kelvin, $T_{0}$ is satellite brightness temperature of IRS 4 in Kelvin, and $T_{a}$ is the mean atmospheric temperature in Kelvin. $\varepsilon$ is ground emissivity while $\tau$ is atmospheric transmittance. $a$ and $b$ are the coefficients that can be approximated according to the following equation:

$$
G=a+b T_{0}
$$

where $G$ is a temperature variable. For IRS4, $G$ has a higher relationship with $T_{0}$. Since the possible temperature range is $0^{\circ} \mathrm{C}$ to $55^{\circ} \mathrm{C}$ in most cases, the coefficients in (3) and (4) were approximated as $a=-69.158, b=0.4684$ with a correlation coefficient $R^{2}=0.9997$ according to IRS4 channel response function published by the China Center for Resources Satellite Data and Application. $T_{0}$ is calculated according to the following equation:

$$
T_{0}=\frac{K_{2}}{\ln \left[1+K_{1} / L\right]},
$$

where $L$ is the radiance that can be calculated by (2). $K_{1}$ and $K_{2}$ are prelaunch calibration constants. For IRS4, which was 
used in this study, $K_{1}=589.33 \mathrm{~W} \cdot \mathrm{m}^{-2} \cdot \mathrm{sr}^{-1} \cdot \mu \mathrm{m}^{-1}$ and $K_{2}=$ $1249.91 \mathrm{~K}$.

There are many ways to calculate the ground emissivity. In this paper, the methods of Normalized Difference Vegetation Index (NDVI) threshold and fractional vegetation cover (FVC) are combined to estimate the $\varepsilon$ for each pixel. NDVI and $\mathrm{FVC}$ are calculated according to the following equation:

$$
\begin{aligned}
\mathrm{NDVI} & =\frac{(\text { Band } 4-\mathrm{Band} 3)}{(\text { Band } 4+\mathrm{Band} 3)}, \\
P_{V} & =\left[\frac{\left(\mathrm{NDVI}-\mathrm{NDVI}_{S}\right)}{\left(\mathrm{NDVI}_{V}-\mathrm{NDVI}_{S}\right)}\right]^{2},
\end{aligned}
$$

where band 3 and band 4 are reflectance values in the red region and NIR of HJ-1B CCD images. $P_{V}$ is fractional vegetation cover. $\mathrm{NDVI}_{V}$ is the minimum NDVI value of pure vegetation pixel, and $\mathrm{NDVI}_{S}$ is the maximum NDVI value of pure soil pixel.

In order to preadjust calculation, typical substance emissivity such as vegetation, soil, water, and impervious surface is generally included in the calculation formula. The vegetation emissivity values are located between 0.98 and 0.99 , and water emissivity is usually considered as 0.995 based on previous research findings [56]. The impervious surface emissivity is selected between 0.960 and 0.980 , and soil emissivity is usually selected between 0.970 and 0.980 [57]. For the pure vegetation pixel of the $\mathrm{HJ}-1 \mathrm{~B}$ image, we selected the vegetation emissivity $\varepsilon_{V}=0.986, P_{V}=1$ when NDVI $\geq$ $\mathrm{NDVI}_{V}$. For the pure soil pixel, we selected the soil emissivity $\varepsilon_{S}=0.972, P_{V}=0$ when NDVI $\leq \mathrm{NDVI}_{S}$. For the pure water pixel, we selected the water emissivity $\varepsilon_{W}=0.995$, while for the impervious surface emissivity $\varepsilon_{M}=0.968$. Vegetation, soil, water, and impervious surface emissivity values were based upon the local conditions and the substance's spectrum curve. For the mixed pixel of soil and vegetation, $\varepsilon$ can be calculated according to (7). For the mixed pixel of impervious surface area and vegetation, $\varepsilon$ can be calculated according to (8):

$$
\begin{aligned}
& \varepsilon=P_{V} R_{V} \varepsilon_{V}+\left(1-P_{V}\right) R_{S} \varepsilon_{S}+d_{\varepsilon}, \\
& \varepsilon=P_{V} R_{V} \varepsilon_{V}+\left(1-P_{V}\right) R_{m} \varepsilon_{m}+d_{\varepsilon},
\end{aligned}
$$

where $d_{\varepsilon}$ is error in emissivity value. It is the mean weighted value that can be calculated by the mean emissivity value of different surface types. Weighted value $d_{\varepsilon}=0.04$ (approximation) was adopted in this paper. $R_{V}, R_{S}$, and $R_{m}$ are temperature ratios of vegetation, bare soil, and impervious surface area, respectively. Qin et al. (2004) presented an elaborate determination of the ratio for accurate LST retrieval from Landsat TM6 data and gave the following accurate estimation of the ratio from the fractional vegetation of pixel [58]:

$$
\begin{gathered}
R_{V}=0.9332+0.0585 P_{V}, \\
R_{S}=0.9902+0.1068 P_{V}, \\
R_{m}=0.9886+0.1287 P_{V} .
\end{gathered}
$$

Duan et al. established an equation for the relationship between atmospheric transmittance $\tau$ and water vapor content $\omega$ for IRS 4 as follows [59]:

$$
\begin{aligned}
\tau & =0.9821-0.1241 \omega \quad\left(R^{2}=0.967\right) \\
\omega & =\left\{\frac{[\alpha-\ln (\rho 19 / \rho 2)]}{\beta}\right\}^{2},
\end{aligned}
$$

where $\alpha$ and $\beta$ are constants taken as 0.02 and 0.651. $\rho 2$ and $\rho 19$ are reflectance values in band 2 and band 19 of MODIS, due to the fact that bands 2 and 19 are sensitive to atmospheric water vapor and that the satellite overpass time for MODIS and $\mathrm{HJ}-1 \mathrm{~B}$ is very close.

The last parameter in (3) is the mean surface temperature $T_{a}$. Duan proposed four models of different atmospheric profiles for calculating $T_{a}$ of IRS4 [51]. Since Shenzhen city lies in the tropics, the tropic model was adopted to calculate $T_{a}:$

$$
T_{a}=22.13009+0.89852 T_{0} .
$$

The tropic model has been successfully applied for LST retrievals by previous researchers [53]. All analysis was completed using the software ENVI 5.3. After the completion of the above steps, the LST images were obtained as shown in Figure 2.

2.4. Comparison with MODIS LST Product. Multiple daily LST products have been used in urban thermal environment studies worldwide. The data are generated by the science team of the Moderate Resolution Imaging Spectroradiometer (MODIS) onboard the NASA Terra and Aqua Earth Observation System satellites. In this paper, MODIS daily temperature products (MOD11A1) provided by NASA were employed to validate the results of LST retrieved from HJ-1B. MOD11A1 data consist of level 3 daily LST products acquired at $1 \mathrm{~km}$ resolution which have been extensively used in SUHI monitoring studies $[60,61]$. The split-window algorithm is used to retrieve LST by applying multiple bands from the $31(10.78 \mu \mathrm{m}-11.28 \mu \mathrm{m})$ and 32 band $(10.77 \mu \mathrm{m}-12.27 \mu \mathrm{m})$ of MODIS. The accuracy of the MOD11A1 has been verified and the margin error is $1.0 \mathrm{~K}$ [62]. Previous studies have proven MOD11A1 is the useful tool for experts and scholars to validate the accuracy of the LST retrieved from HJ-1B $[63,64]$.

The MOD11A1 data, whose overpass time is close to that of $\mathrm{HJ}-1 \mathrm{~B}$ satellite on the same day, were geometrically corrected using NASA's MRT tool. The MODIS LST $\left({ }^{\circ} \mathrm{C}\right)$ for Shenzhen can be calculated as follows [65]:

$$
T_{S}=\mathrm{DN} \times 0.02-273.15,
$$

where $T_{S}$ is the LST value and DN is the pixel gray value. The radiation scaling ratio is 0.02 , as indicated in MOD11A1 products' header files. The radiation scaling distance is 0 and 273.15 is the difference value between Kelvin temperature $(\mathrm{K})$ and Celsius degree $\left({ }^{\circ} \mathrm{C}\right)$.

In order to match the images, MOD11Al data were resampled to pixels of $300 \mathrm{~m} \times 300 \mathrm{~m}$ for comparison 


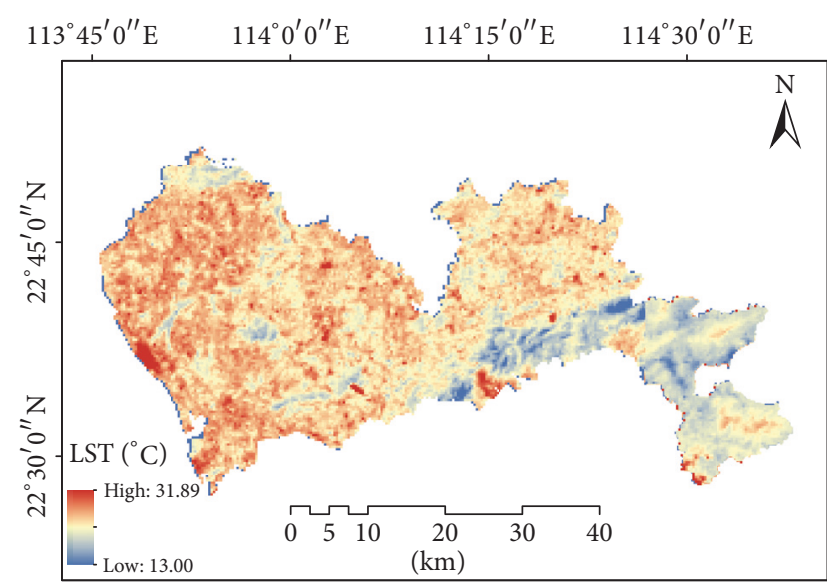

(a) $2015-01-16$ (winter)

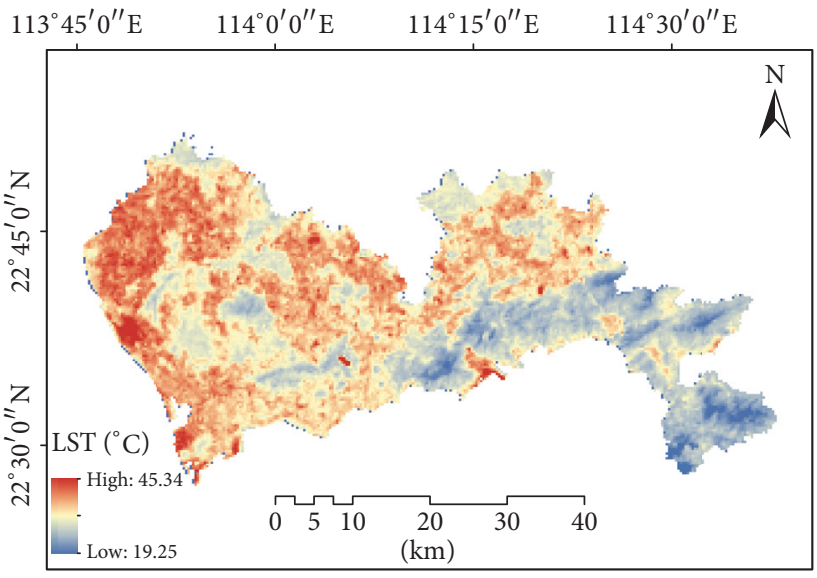

(c) $2015-10-18$ (summer)

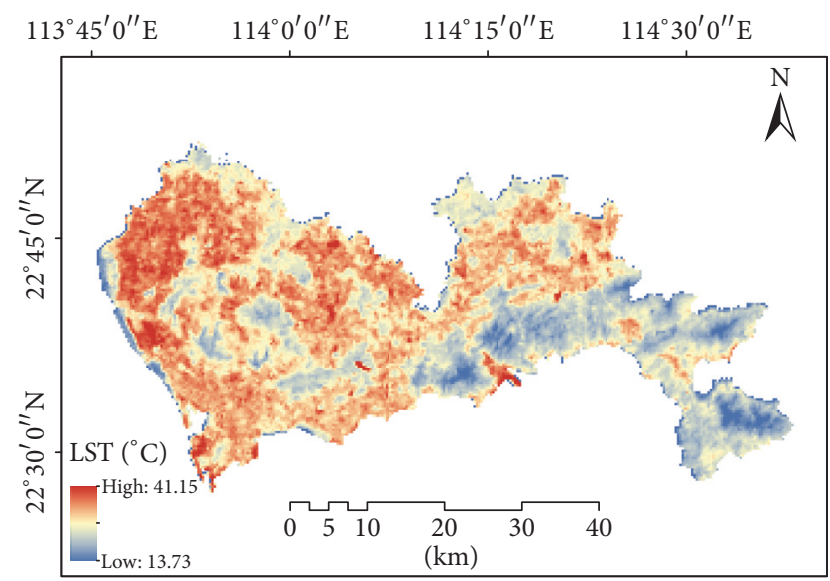

(b) 2015-04-14 (spring)

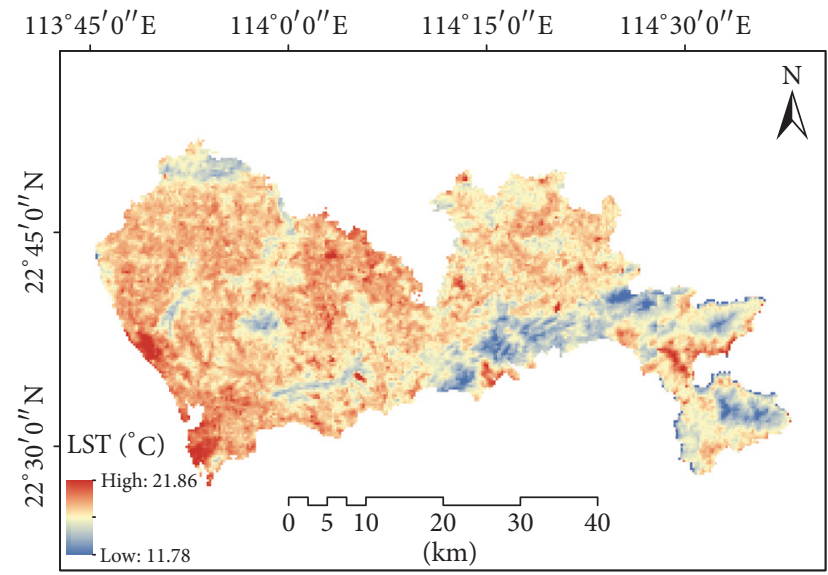

(d) 2015-12-18 (autumn)

FIGURE 2: Spatial distribution of LST in Shenzhen from spring through winter, 2015.

with LST retrieved from HJ-1B for the same study period. Since the temperature distribution over the lake surface is homogeneous, Xiangmi Lake, Shenzhen Reservoir, Yantian Reservoir, Meilin Reservoir, and Xili Reservoir were selected in study area as the ground target for verification. Then 100 sampling points over lake surfaces were randomly selected for accuracy assessment, and the results are given in Table 4. By comparison, it can be concluded that the inversion accuracy of LST based on HJ-1B thermal infrared data is high, and the correlation coefficients were $0.873,0.859,0.877$, and 0.815 in winter, spring, summer, and autumn, respectively, and had passed $T$ test using a significance level of 0.01 . Moreover, the error indicators of RMSE and MAE are less than 3 and 2, respectively. All of this indicated that LST retrievals from $\mathrm{HJ}$ $1 \mathrm{~B}$ can be trusted in this area.

2.5. Determination of Urban Heat Island Intensity. Urban heat island intensity (UHII) is an important indicator to evaluate the severity of SUHI [65]. In this study, UHII is defined as the difference between the LST of each pixel and the mean surface temperature in $\mathrm{HJ}-1 \mathrm{~B}$ images [66]. The approach tends to differ from the other regional or global UHII analyses that took "rural" area (a certain distance away from urban) as reference locations $[67,68]$, since Shenzhen is the first city in China with no rural administrative system. There is no rural social system and the urbanization rate amounts to $100 \%$. Since 2003, the government passed a series of regulations to urbanize all rural areas of Shenzhen [69] and took over all local rural residents' owned land after attributing an urban resident status to all rural residents of that time. As most of rural areas were surrounded by the city's built-up centers, these changes not only turned all traditional villages into "urban villages," but also marked the end of the urban-rural system division in Shenzhen. It can therefore be stated that there is not a reasonably large "rural area" to be considered as a reference and it is practically impossible to demarcate the boundaries between the city and the rural area.

The UHII calculation method adopted here can reduce the uncertainties associated with site-specific rural conditions across Shenzhen city (e.g., topography, the presence of water body, and land use). It can be calculated as follows:

$$
\mathrm{UHII}_{i j}=T_{i j}-\overline{T_{a}}
$$


TABLE 4: Comparisons of LST estimates retrieved from HJ-1B and MOD11A1.

\begin{tabular}{|c|c|c|c|c|c|c|c|c|}
\hline \multirow{3}{*}{ Parameter } & \multicolumn{8}{|c|}{ Date } \\
\hline & \multicolumn{2}{|c|}{ 2015.1.16 } & \multicolumn{2}{|c|}{ 2015.4.14 } & \multicolumn{2}{|c|}{2015.10 .18} & \multicolumn{2}{|c|}{ 2015.12.18 } \\
\hline & $\mathrm{HJ}-1 \mathrm{~B}$ & MOD11 & $\mathrm{HJ}-1 \mathrm{~B}$ & MOD11 & $\mathrm{HJ}-1 \mathrm{~B}$ & MOD11 & $\mathrm{HJ}-1 \mathrm{~B}$ & MOD11 \\
\hline RMSE values & \multicolumn{2}{|c|}{1.374} & \multicolumn{2}{|c|}{2.013} & \multicolumn{2}{|c|}{1.756} & \multicolumn{2}{|c|}{1.970} \\
\hline MAE values & \multicolumn{2}{|c|}{0.892} & \multicolumn{2}{|c|}{1.743} & \multicolumn{2}{|c|}{1.521} & \multicolumn{2}{|c|}{1.692} \\
\hline Correlation coefficient & \multicolumn{2}{|c|}{0.873} & \multicolumn{2}{|c|}{0.859} & \multicolumn{2}{|c|}{0.877} & \multicolumn{2}{|c|}{0.815} \\
\hline
\end{tabular}

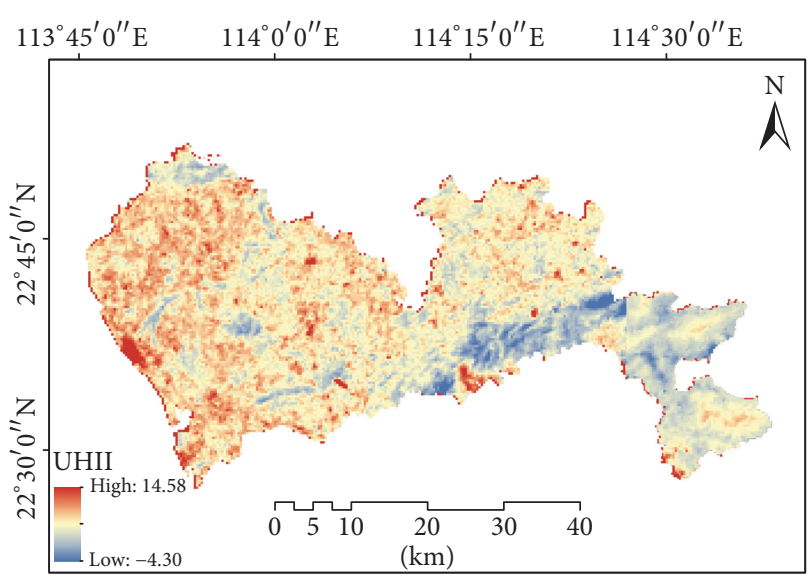

(a) 2015-01-16 (winter)

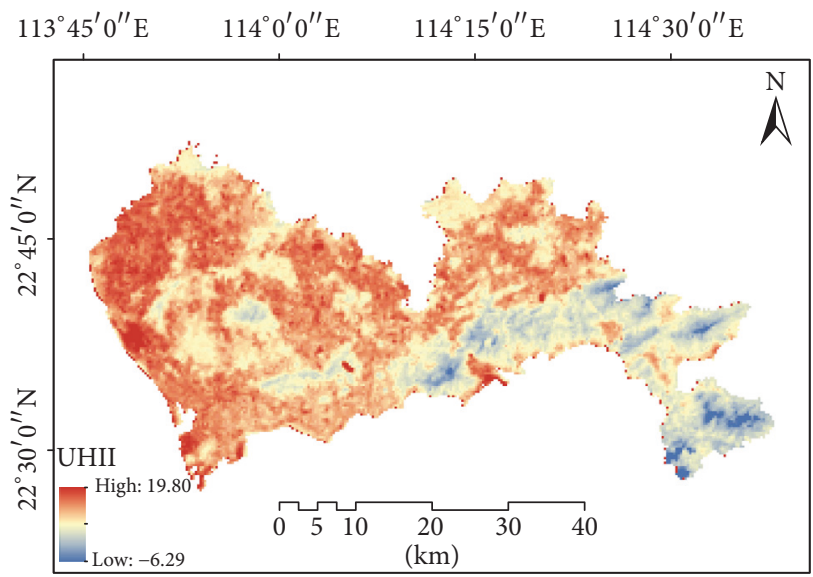

(c) $2015-10-18$ (summer)

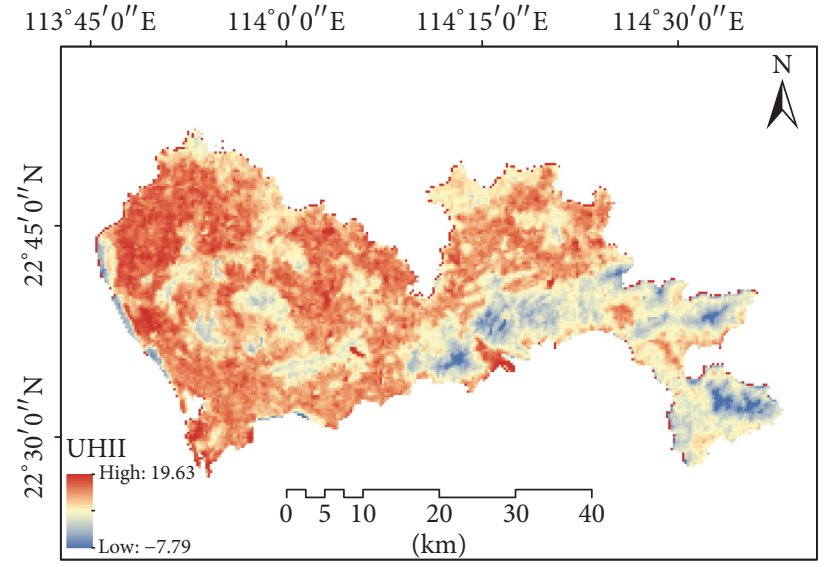

(b) 2015-04-14 (spring)

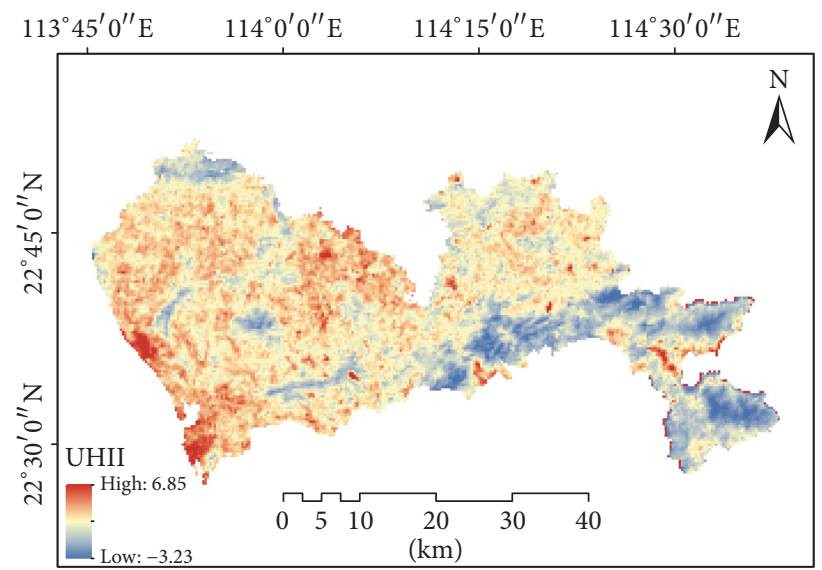

(d) 2015-12-18 (autumn)

FIgURE 3: Spatial distribution of UHII in Shenzhen from spring through winter, 2015.

where $\mathrm{UHII}_{i j}$ and $T_{i j}$ are SUHI intensity and LST of pixels $i, j$ in HJ-1B image, respectively. $\overline{T_{a}}$ is mean surface temperature in the image (Figure 3).

2.6. Classification of LST Level. The method of the density segmentation was used to classify the urban surface thermal environment after LSTs were normalized, which can reduce atmospheric and calibration correction errors $[31,70]$.

Firstly, LST values were normalized between 0 and 1 during different seasons in 2015. The normalized value was calculated using

$$
N_{i}=\frac{\left(T_{i}-T_{\min }\right)}{\left(T_{\max }-T_{\min }\right)},
$$

where $N_{i}$ is the normalized LST value of the pixel $i, T_{i}$ is the LST of the pixel $i$, and $T_{\max }$ and $T_{\min }$ are the maximum and minimum LST over the entire study area.

To reflect the spatiotemporal distribution of LST directly, the normalized LST value was further divided into seven levels: very high, high, sub-high, medium, sub-medium, low, and very low. The classification criteria of LST level are listed in Table 5.

2.7. Spatial Autocorrelation Analysis. Spatial autocorrelation is an indicator which measures the aggregation degree of the spatial attribute value [71]. It mainly reflects the relevance of the same variable in different spatial positions. The global autocorrelation analysis has been widely used to detect the 
TABLE 5: The classification criteria of LST level.

\begin{tabular}{lc}
\hline LST level & Range \\
\hline Very high & $N_{i} \geq T_{\text {mean }}+2.5 s$ \\
High & $T_{\text {mean }}+1.5 s \leq N_{i}<T_{\text {mean }}+2.5 s$ \\
Sub-high & $T_{\text {mean }}+0.5 s \leq N_{i}<T_{\text {mean }}+1.5 s$ \\
Medium & $T_{\text {mean }}-0.5 s \leq N_{i}<T_{\text {mean }}+0.5 s$ \\
Sub-medium & $T_{\text {mean }}-1.5 s \leq N_{i}<T_{\text {mean }}-0.5 s$ \\
Low & $T_{\text {mean }}-2.5 s \leq N_{i}<T_{\text {mean }}-1.5 s$ \\
Very low & $N_{i}<T_{\text {mean }}-2.5 s$ \\
\hline
\end{tabular}

Note. $T_{\text {mean }}$ is the mean LST value of all pixels after normalization and $S$ is standard deviation.

spatial distribution of UHII value in the study area. One of the most commonly used methods is Moran's $I$ index [72]. In this paper, Moran's I index was utilized to analyze the UHII of Shenzhen city in order to reveal the aggregation degree of the UHII value in the study area. Moran's $I$ value may be negative or positive, representing a negative or positive spatial autocorrelation, respectively. When Moran's $I$ value approaches 0 , it represents the values of spatial autocorrelation near the mean or simply indicates the nonexistence of spatial autocorrelation. Moran's I was calculated using (15) as follows:

$$
\begin{aligned}
& I=\frac{\left[n \sum_{i=1}^{n} \sum_{j=1}^{n} C_{i j}\left(x_{i}-x\right)\left(x_{j}-x\right)\right]}{\left[\sum_{i=1}^{n} \sum_{j=1}^{n} C_{i j} \sum_{i=1}^{n}\left(x_{i}-x\right)^{2}\right]}, \\
& x=\frac{1}{n} \sum_{i=1}^{n} x_{i},
\end{aligned}
$$

where $I$ is Moran's $I$ index value, $x_{i}$ is the attribute of the pixel of $i$ in UHII image, $x$ is the mean UHII change and can be calculated using (16), $n$ is the total number of observations, and $C_{i j}$ is the spatial weight matrix and can represent the relationship between data sites $i$ and $j$ in spatial attributes of UHII.

2.8. Gravity Center Model. Based on the change trend analysis of UHII, centroid moment analysis of UHII was conducted to reflect the dynamics of the urban thermal environment patterns in Shenzhen for different seasons. That is to say, the center of gravity is an important indicator which can describe the spatial distribution and transition of UHII. It not only shows the tendency of spatial distribution, but also reflects the "high-density" parts and overall heterogeneity [73]. In this paper, the gravity center model was used to reflect the overall transfer trajectory of the distribution of UHII during different seasons in Shenzhen. It has a great potential to reveal the evolution of seasonal SUHI in the study area. For UHII geographical objects, the coordinates of gravity center were computed using the following equation:

$$
\begin{gathered}
X_{G}=\frac{\sum_{i=1}^{n} A_{i} x_{i}}{\sum_{i=1}^{n} A_{i}}, \\
Y_{G}=\frac{\sum_{i=1}^{n} A_{i} y_{i}}{\sum_{i=1}^{n} A_{i}},
\end{gathered}
$$

where $X_{G}$ and $Y_{G}$ are the centroid coordinates of UHII calculated by area-weighted average, $x_{i}$ and $y_{i}$ are the centroid coordinates of pixels $i$ with UHII, $A_{i}$ is the area of pixels $i$, and $n$ is the number of all pixels with the same UHII value.

\section{Results}

3.1. Spatiotemporal Distribution Dynamics of LST. LSTs in Shenzhen during different seasons of the year 2015 were quantitatively retrieved from $\mathrm{HJ}-1 \mathrm{~B}$ data, and their spatiotemporal distribution was further analyzed (Figure 2). In order to reflect the spatial variations of LST during four seasons, we used the detailed land use reference map derived from the high resolution $(17 \mathrm{~m})$ Google Earth image dated on April 14, 2015. The supervised maximum likelihood classification method was used to obtain the land use reference map composed of seven land use/land-cover types, notably impervious surfaces area (ISA), forest land, public gardens, plough land, grass land, unused land, and water bodies as shown in Figure 4.

Spatially, LSTs showed a decreasing trend from west to east, and the high temperature regions were concentrated in the Western Industrial Cluster, Qianhai and Futian-Luohu Urban Municipal Center, Central Urban Cluster, and Eastern Industrial Cluster. Several reasons may be attributed to occurrence of higher temperatures in the abovementioned regions. Firstly, the Western Industrial Cluster is close to the cities of Guangzhou and Dongguan, having a large number of ports, airports, factories, universities, and residential areas, and has become the most important base for high-tech and manufacturing industries in the Pearl River Delta region. Secondly, Qianhai and Futian-Luohu Urban Municipal Center is a gigantic business center, established on the development of corridors of Guangzhou and Hong Kong and serves as an international production service center. There are many urban villages, old residential and industrial areas. Finally, the Central Urban Cluster has become the major site for real estate development due to its location advantages. Eastern Industrial Cluster has also had rapid industrial development underpinned by government efforts to promote industrial expansion in the area. Besides, SUHI effect is not obvious in Dapeng Peninsula (southeastern Shenzhen) whose terrain is largely mountainous along the coast. This region is also adorned by a significant number of water bodies and green areas that substantially attenuate thermal radiations. Areas with the low LSTs also include Xili Street in the southwest of the city and Longgang Center Cluster in the central part of the city owing to the presence of numerous water reservoirs such as lakes and a hilly topography. The highest surface temperatures were found in the important transportation hubs, such as Baoan International Airport in the west, Shekou Port in the southwest, and Yantian Port in the south.

The seasonal trends of LST are shown in Figure 2; in winter, LST values ranged from 13 to $31.89^{\circ} \mathrm{C}$ with higher surface temperatures mainly concentrated in business and industrial districts of Nanshan, Baoan, Longgang, and Guangming. SUHI effect was strikingly more pronounced in spring and summer where the difference between the peak value and the lowest value would go as high as $27.42^{\circ} \mathrm{C}$. Apparently, two 


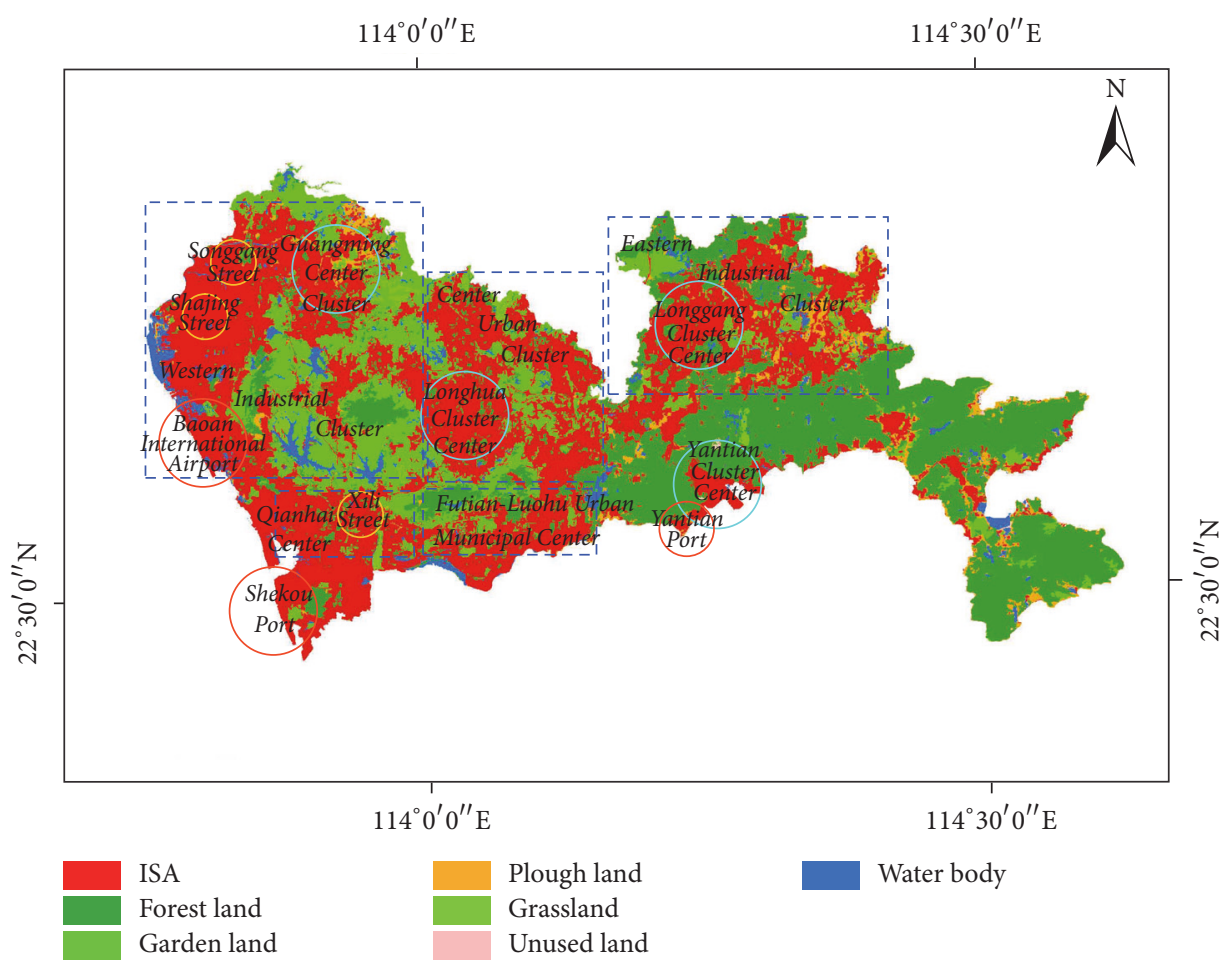

FIgURE 4: Detailed land use reference map in Shenzhen, 2015.

hot areas can be identified: Western Industrial Cluster and Central Urban Cluster, especially in industrial concentration areas of Shajing and Songgang Street, and traditional manufacturing, commercial, and residential concentration areas of Guangming District. The autumn LST values ranged from $11.78^{\circ} \mathrm{C}$ to $21.86^{\circ} \mathrm{C}$ and had a mean value of $15.01^{\circ} \mathrm{C}$ (Table 4 ). The highest temperatures were in the Qianhai and FutianLuohu Urban Municipal Center where urbanization is at its peak together with the political, business, high-end services and residential functions. Generally, the LST values were higher during summer and spring compared with winter and autumn. In all seasons, the LST was highest in transportation hubs followed by commercial areas, industrial areas, and residential areas, while the lowest occurred in green areas and water body areas with LST of $11.78^{\circ} \mathrm{C}$ in autumn and $19.25^{\circ} \mathrm{C}$ in summer, respectively. The reasons for seasonal variations in LSTs values within the study area are very clear. All higher LST values were located in commercial activities' centers and principal residential zones, while all lower LST values areas consisted of vegetation and water covers [74].

\subsection{Seasonal Variation of UHII and Its Profiles Characteristics.} Figure 3 displays the spatial distribution of UHII in Shenzhen during spring through winter in 2015. It can be observed that UHII values are higher in spring and summer and lower in autumn and winter. The red area with higher UHII intensity is vastly concentrated in spring and summer, while a lower UHII intensity and more even distribution have been noted in autumn and winter. The UHI has distinctive characteristics according to regions in conformity with the trajectory of urban expansion.
Based on "The 2010-2020 Comprehensive Plan of Shenzhen City," five profiles (Figure 5(a)) were drawn to analyze the distribution of the UHII. The No. 1 path profile (Figure $5(\mathrm{~b}))$ is in the western developmental axis whose main function is to develop modern service and manufacturing industry. It started with Qianhai Center Cluster and went alongside the Pearl River to connect the Baoan District and the Western Industrial Cluster, ultimately linking it with the city of Guangzhou. The No. 2 path profile (Figure 5(c)) is the central developmental axis whose main function is to develop integrated services, high-tech industries, and advanced manufacturing. The profile sets out at FutianLuohu Urban Municipal Center and passes through Longhua and Guangming Center Cluster, before reaching the Dongguan city. The No. 3 path profile (Figure 5(d)) lies in the eastern developmental axis whose main function is to develop high-tech industries and advanced manufacturing. The profiles begin at the Futian-Luohu Urban Municipal Center and successively traverses the Buji and Longgang Center Cluster before ending in the Huizhou city. The No. 4 path profile (Figure 5(e)) is the northern developmental belt with a function of developing multifunctional industries. It started with Hong Kong Town and went alongside the railways and highways to connect Longhua, Longgang, and Pingshan Center Cluster, ultimately linking to Huizhou and Shantou cities. The No. 5 path profile (Figure $5(\mathrm{f})$ ) is the southern developmental belt whose purpose was to boost finance and tourism due to its favorable emplacement along the development corridors of Guangzhou and Hong Kong. The profile sets off at Qianhai Center, passes through FutianLuohu Urban Municipal Center, and arrives at the Daya Bay. 

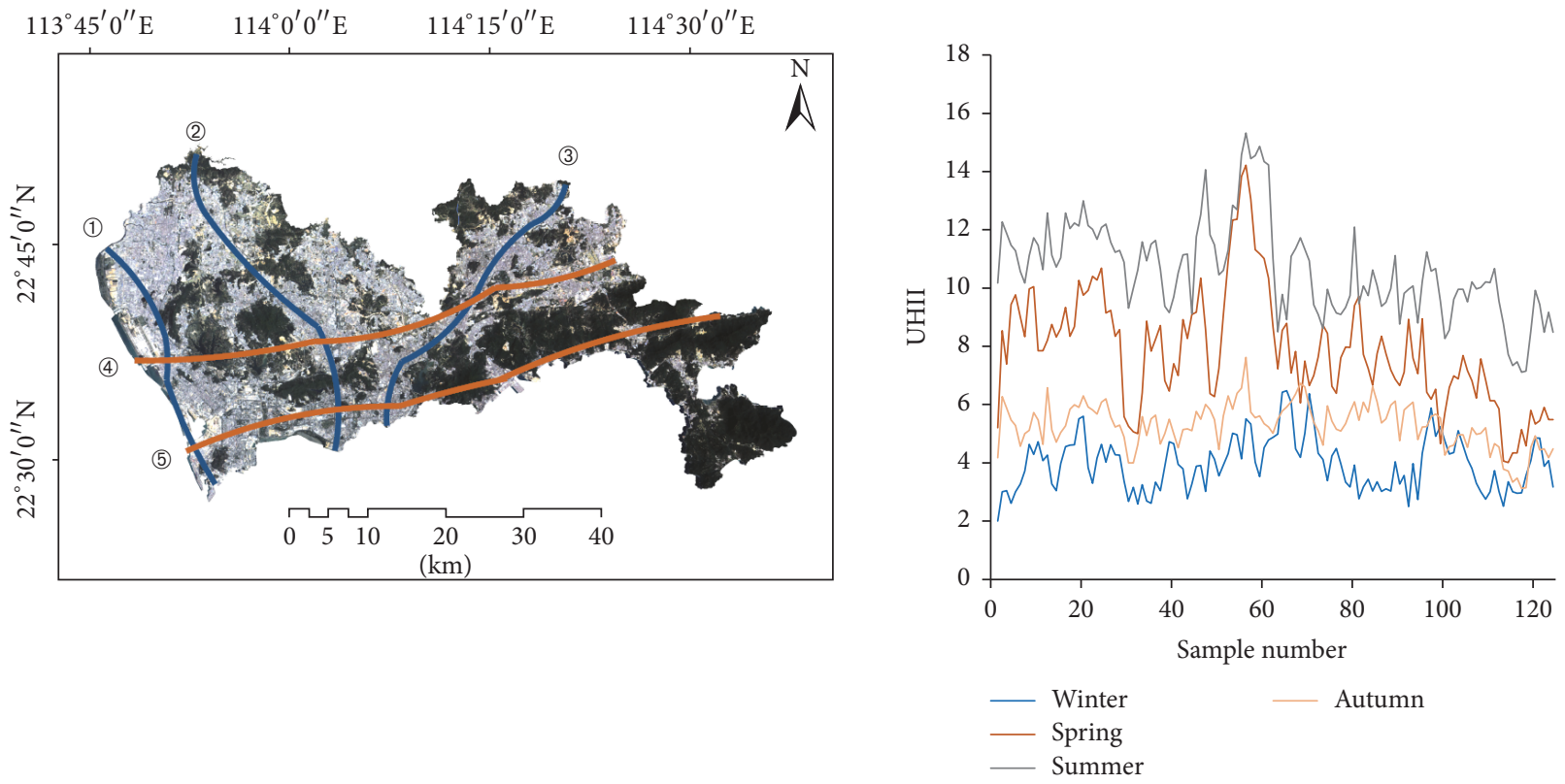

(a) Sketch map of UHII in sample path

(b) Profiles of UHII in sample path (1)

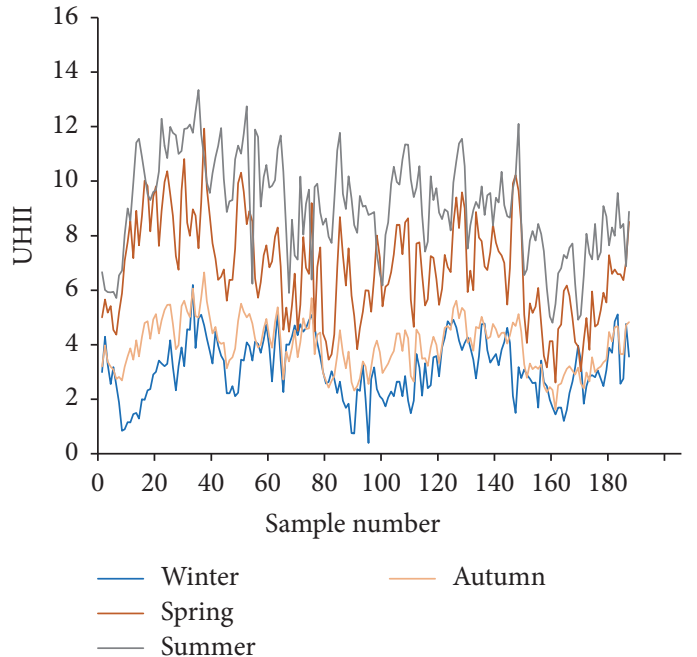

(c) Profiles of UHII in sample path (2)

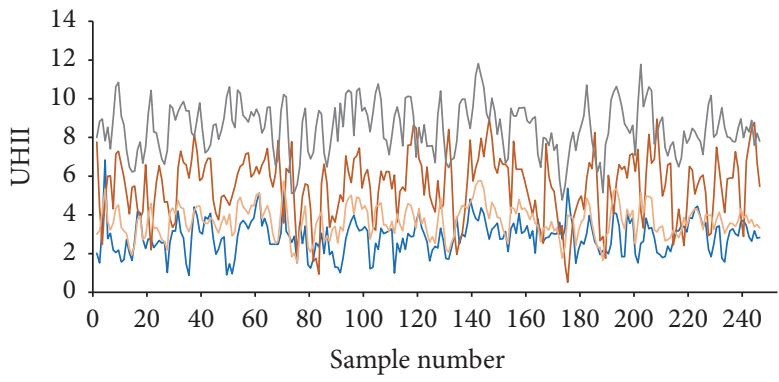

(e) Profiles of UHII in sample path (4)

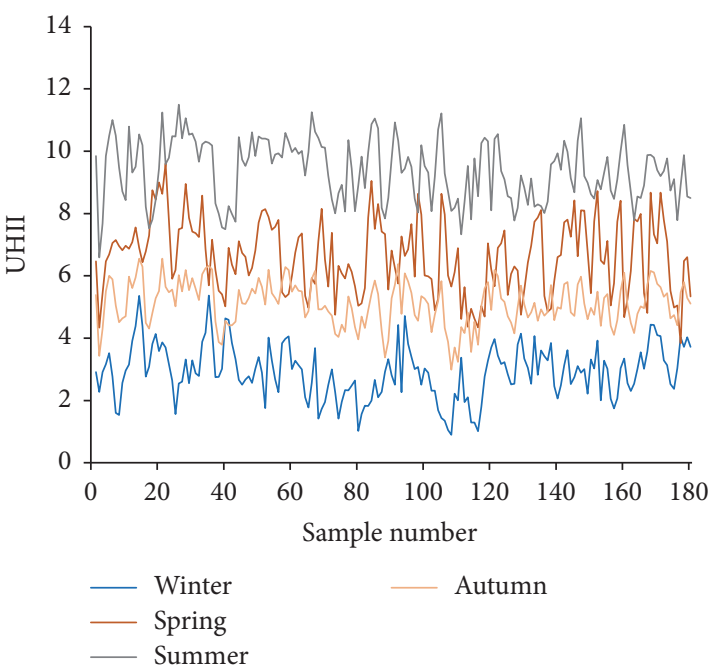

(d) Profiles of UHII in sample path (3)

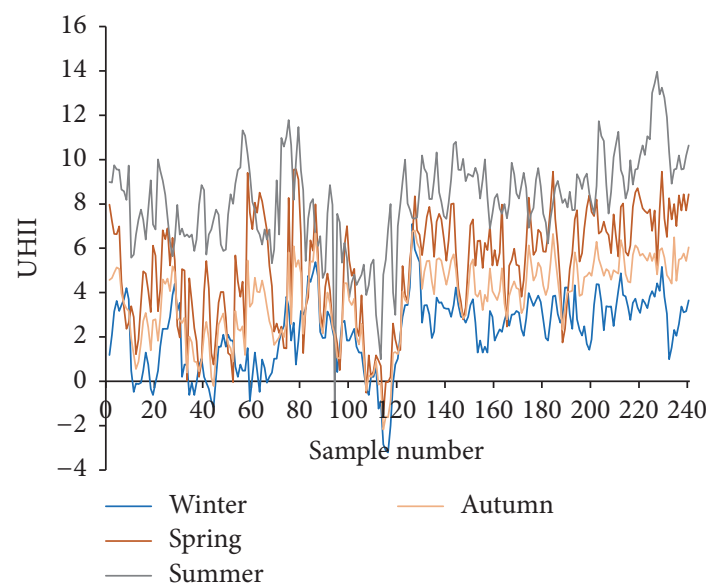

(f) Profiles of UHII in sample path (5)

FIGURE 5: Differences in UHII of different sample path profiles from spring through winter, 2015. 
Figure 5 displays all sample paths of UHII profiles in Shenzhen from spring through winter in 2015. From the city's No. 1 to No. 5 path profiles, there exist numerous "peaks," "basins," and "plateaus," indicating the heterogeneous nature of UHII over the area and also reflecting the influence of different types of land use and occupation on the UHII along the profile paths. Many factors such as the occurrence of mountains, other green spaces, water bodies, high population densities, buildings, and the administrative subdivision of the city's functional districts may exert an influence on the spatial distribution of UHII. The seasonal variation of UHII becomes visible when comparing the different sample paths of UHII profiles in Figure 5. It appears that UHII value varies according to seasons. The highest value dimension belongs to summer, followed by spring and autumn, while the lowest value dimension is found in winter. The effect of UHII becomes more prominent when the season changes from autumn to spring. The No. 1 path profiles have a higher value of UHII than other path profiles. This indicates that No. 1 path profiles cross more built-up or impervious areas that possess higher thermal signatures than the other path profiles. Indeed, the No. 1 path profiles pass over the area with the highest impervious surface coverage such as airports, ports, and industries. The relatively low value of UHII was found in the No. 5 path profiles, most likely because this path crosses more land-cover types, encompassing water bodies and greenspaces. It can be noted in Figure 5 that, in almost all paths in winter, the UHII values were lower than in other seasons, while, in summer, most UHIIs were at their highest. The values of UHII in summer and spring are relatively close, while the UHII values in winter and autumn are relatively close. There is a contrast, therefore, especially in the winter and summer seasons. Over some water bodies particularly, the UHII value goes from zero to negative. This profile is an important ecological belt established by Shenzhen municipality authorities to protect the environment, containing many mountains, water bodies, parks, beaches, and green tourist attractions. Impervious surfaces and built-up areas occupy a nonsignificant portion of the land.

3.3. Spatiotemporal Distribution of the LST Levels. Based on the significance of LST level classification (Figure 6), the spatiotemporal diversity of LST in Shenzhen can be disentangled through anomaly analysis to determine "high temperature zones" (including very high, high, and subhigh), "medium temperature zones," and "low temperature zones" (including sub-medium, low, and very low). High temperature zones occupy the largest proportion in spring and summer, and the medium temperature zones occupy the largest proportion in autumn and winter.

Analysis of seasonal temperature has revealed that, in autumn and winter, "medium temperature zones" occupy a large proportion, followed by "low temperature zones" whereas "high temperature zones" account for the smallest proportion. By contrast, in spring and summer, "low temperature zones" occupy a large proportion, followed by "high temperature zones" and "medium temperature zones." The "high temperature zones" in spring and summer were centered in the Western Industrial Cluster, indicating a high
UHII. The southeast of Shenzhen is covered by mountains and the vegetation is very dense during spring and summer, which may explain the relatively low UHII. During winter and autumn, "high temperature zones" are few and extremely scattered. However, "very high temperature zones" were detected in all seasons and were concentrated over the Baoan International Airport, Shekou Port, and Yantian Port, underscoring the strong impact of anthropogenic activities on urban thermal environment.

3.4. Spatial Autocorrelation Analysis. Moran's I index was used to perform spatial autocorrelation analysis (Figure 7). Moran's $I$ index value ranges between -1 and 1 . When the value is larger than 0 , the autocorrelation is positive. When the value is lower than 0 , there is a negative correlation in the spatial patterns. When the value is 0 , it means that there is no spatial correlation. In this study, Moran's $I$ index values in different seasons are all above 0.5 , indicating that there was a positive spatial autocorrelation for UHII in different seasons in this study. Higher values in summer and spring, followed by winter and autumn, suggest that the aggregated change between high and low temperature zones was more apparent in summer and spring.

\subsection{Gravity Center of UHII Shifts during Different Seasons.} The center of gravity of UHII shifts was concentrated in Longhua District, Baoan District, and Nanshan District throughout the four seasons (Figure 8). This is because Longhua District belongs to the Central Urban Cluster, Baoan District belongs to the Western Industrial Cluster, and Nanshan District shelters the Qianhai Municipal Center. In winter, UHII gravity center is primarily located in Longgang District due to the presence of large numbers of commercial, residential, and public buildings. In spring and summer, UHII gravity center is transferred to the south and the center of Baoan District, indicating that UHII gravity center is controlled by the presence of intensive manufacturing factories and vibrant economic activities such as airports where population and traffic volume are high. In autumn, UHII gravity center shifts towards the Qianhai Municipal Center which is both an international production service center and a grand business center.

\section{Discussion}

4.1. Analysis of Seasonal Variations of Urban Thermal Environment. This paper shows that the analysis of seasonal variations of SUHI is crucial to describe the urban thermal environment in Shenzhen. Selected as the study area, Shenzhen is appropriate for urban thermal environment studies owing to its rapid urbanization characteristics.

The spatial variation characteristics highlighted in this paper are consistent with previous studies that used thermal infrared data, such as Landsat TM/ETM. In 2013, for instance, Xie et al. [75] derived land surface temperature from Landsat TM to investigate the relationship between landscape patterns and LST in Shenzhen and showed that SUHI was located in transportation centers and industrial hubs (especially in Baoan Airport, Qianhai and Yantian Port, and Songgang and Shajing). They argued that ISA can contribute to the 


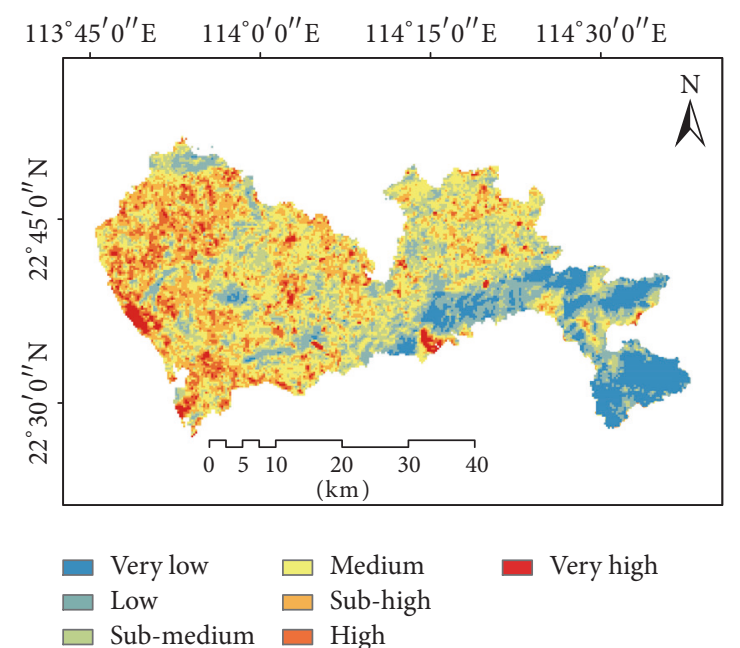

(a) 2015-01-16 (winter)

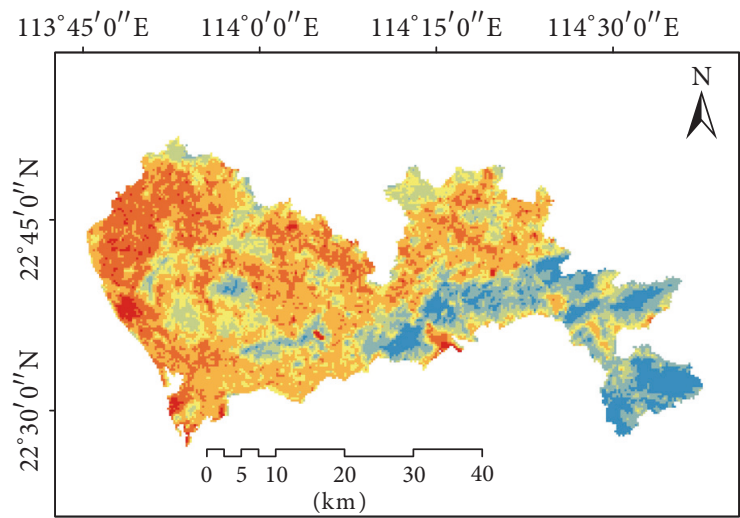

$$
\begin{array}{ll}
\square \text { Very low } & \square \text { Medium } \square \text { Sub-high } \\
\square \text { Low } & \square \text { Very high } \\
\square \text { Sub-medium } & \text { High }
\end{array}
$$

(c) 2015-10-18 (summer)

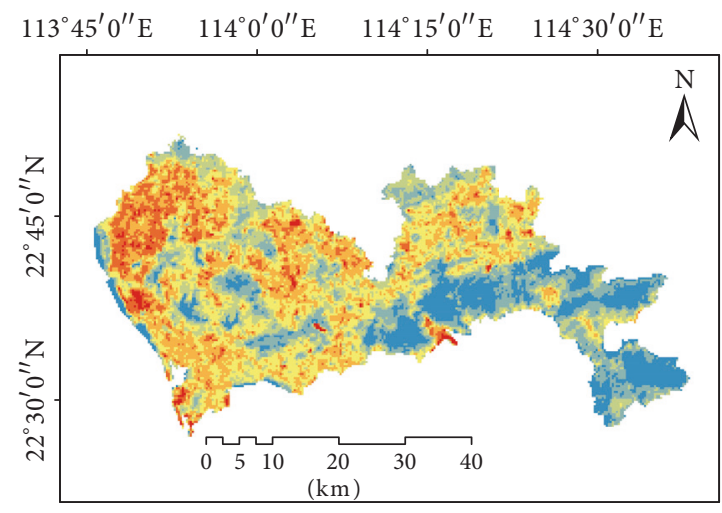

$$
\begin{array}{ll}
\square \text { Very low } & \square \text { Medium } \\
\square \text { Low } & \square \text { Sub-high } \\
\square \text { Sub-medium } & \text { High }
\end{array}
$$

(b) 2015-04-14 (spring)$$
113^{\circ} 45^{\prime} 0^{\prime \prime} \mathrm{E} \quad 114^{\circ} 0^{\prime} 0^{\prime \prime} \mathrm{E} \quad 114^{\circ} 15^{\prime} 0^{\prime \prime} \mathrm{E} \quad 114^{\circ} 30^{\prime} 0^{\prime \prime} \mathrm{E}
$$

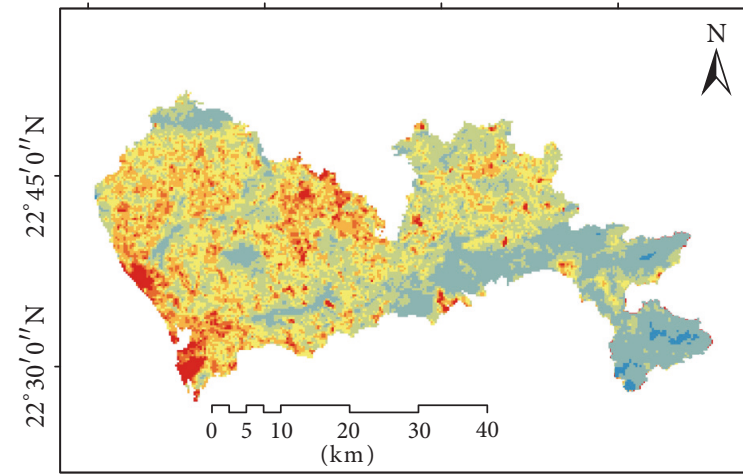

$$
\begin{array}{ll}
\square \text { Very low } & \square \text { Medium } \square \text { Very high } \\
\square \text { Low } & \square \text { Sub-high } \\
\square \text { Sub-medium } & \square \text { High }
\end{array}
$$

(d) 2015-12-18 (autumn)

FIGURE 6: Spatial distribution of LST level in Shenzhen during spring through winter of 2015.

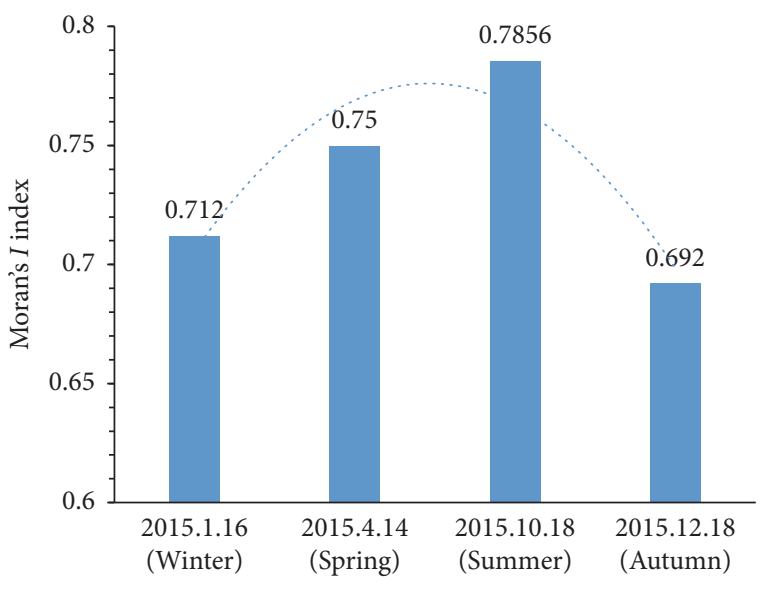

FIgURE 7: Moran's $I$ index of UHII in Shenzhen during spring through winter, 2015. increase of LST and that vegetated areas could contribute to the decrease of LST. Xie et al. [6] also assessed the spatial patterns of the thermal environment in Shenzhen, selecting four profiles for LST distribution analysis, and showed that the peak values in profiles were located in the CBD, industrial land, and transportation centers while low SUHI were located in profiles corresponding to rivers, forests, and lakes.

Several factors may influence the seasonal variation in the SUHI of Shenzhen. Firstly, the differences in surface wind may possibly result in differences in SUHI within four seasons. Lu et al. (2009) found that the urbanization of Shenzhen can have a significant impact on sea breeze [76] and that enhanced wind speed could induce low temperatures. According to the Meteorological Bureau of Shenzhen Municipality, in the published "Shenzhen Climate Report" [77], the average annual wind speed is $2.7 \mathrm{~m} / \mathrm{s}$, and the wind speed value is higher in winter and autumn (monthly mean wind 


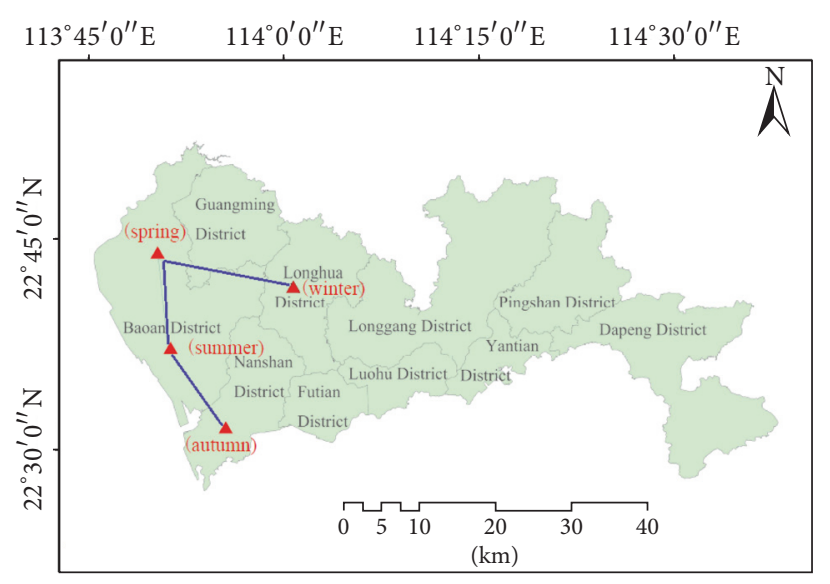

- Gravity center of UHII

- The trajectory of UHII center

FIGURE 8: Change path of UHII gravity in Shenzhen from spring through winter, 2015.

speed within a range of $2.8-3.0 \mathrm{~m} / \mathrm{s}$ ) and lower in summer $(2.1-2.2 \mathrm{~m} / \mathrm{s})$. This is consistent with this study's findings that the values of UHII were higher in spring and summer and lower in autumn and winter. Secondly, the seasonal changes of SUHI were positively and significantly correlated with precipitation [78]. In Shenzhen, summer mean precipitation was $1562.5 \mathrm{~mm}$, followed by spring $(275.4 \mathrm{~mm})$, autumn $(66.0 \mathrm{~mm})$, and winter $(27.7 \mathrm{~mm})$, while the rainfall amount from April to September made up to $84.5 \%$ of the estimated total annual rainfall. Finally, the SUHI was strong in summer and spring whereas it was weak in winter and autumn, possibly due to stronger solar radiation absorbed by urban areas in summer and spring. In Shenzhen, the monthly mean solar radiation value was beyond $400 \mathrm{MJ} \cdot \mathrm{m}^{-2}$ in summer and spring and decreased to $300-400 \mathrm{MJ} \cdot \mathrm{m}^{-2}$ in winter and autumn [77]. Also, stronger human activities in summer and spring may lead to stronger SUHI. It is acknowledged that the seasonal variations of SUHI were related to anthropogenic heat releases [79]. In Shenzhen, human activities such as vehicles, air conditioners, power plants, and other heat sources release a lot of heat, especially in the anthropogenic heat flux for building cooling owing to its tropical maritime climate characterized by long summers and short winters [80].

The results of this study are also congruent with the findings of Qiao et al. [73] who, while studying the influences of urban expansion on urban heat island in Beijing during 1989-2010, found that the UHI gravity center transfer was highly consistent with urbanization patterns and dynamism, although irregular transfers of UHI were observed in some zones. At present, the reasons for shifts in UHII gravity center are still not yet clearly known. This is because UHII center transfers can be affected by a huge number of factors such as vegetation activity [81-83], albedo [84-86], built-up intensity [87], anthropogenic heat emissions [3, 88], and city size and topography $[27,89]$. However, in this study, it has been revealed that seasonal changes have the potential to greatly influence the distribution and transfer of UHII gravity centers.

4.2. Implementation for Urban Planning. SUHI is an important aspect to consider for achieving urban sustainable development and is one of the key levers to solving environmental problems. In light of the above results, three possible mitigating measures can be adopted to counteract SUHI effects in Shenzhen. Firstly, we recommend the reduction of anthropogenic heat release through human activities. A supporting example to that argument is the study conducted by Kikegawa et al. (2003) who estimated that the near-ground temperature decreased by more than $1^{\circ} \mathrm{C}$ by cutting off all air-conditioning waste heat of buildings (discharge of waste heat into media other than the atmosphere) in a central business district in Tokyo [90]. Mirzaei and Haghighat (2010) reported that anthropogenic heat release was the main reason for the SUHI in metropolitan areas [91]. In Shenzhen, as the metropolitan city of China, the increase of energy consumption and huge demand for summer electricity caused SUHI effect to become more and more severe, especially in CBD, industrial areas, and residential areas. Therefore, sources of heat-waste from air-conditioning of buildings should be minimized and anthropogenic heat release should be reasonably controlled to the maximum. Secondly, better roof designs should be adopted. Research has found that the roof surface of the building can be used to reduce the surface temperature of the urban areas since roof surfaces occupy $20-25 \%$ of the total urban surface [92]. Several studies have proved that cool roof and green roof are the two main technologies for mitigating the UHI $[93,94]$. Cool roofs are characterized by materials permitting high thermal emittance and high solar reflectance. These materials can reflect the incident solar radiation away from the building, which keeps their roof surface cooler compared to traditional materials. Green roofs, on the other hand, use the foliage of plants to cover the roof surface. The vegetation and soil can absorb solar radiation and take advantage of the additional thermal insulation [95]. Shenzhen Metropolis is the largest city in China and its high-rise of buildings has become the mainstream of the official and residential developments. Cool and green roofs, as effective SUHI mitigation techniques, can also improve the thermal comfort of not-cooled buildings in Shenzhen [96]. Finally, we underscore the great importance of planting trees and vegetation as it is the most widely applied strategy in mitigating SUHI as elaborated by several studies [97-99].

\section{Conclusions}

This paper investigated the seasonal variation of urban surface thermal environment in Shenzhen during spring to winter of 2015. Remote sensing techniques and GIS spatial analysis tools were used to retrieve LST from HJ-1B data. The indicator of UHII was established and the method of density segmentation was used to classify seven levels of LST values ranging from very high to very low. Spatial analysis including spatial autocorrelation analysis and gravity center moment analysis were carried out to look at the distribution dynamics of SUHI seasonal variation. 
The results showed the following. (1) During the study period, the distribution of LSTs in Shenzhen showed a decreasing trend from the west to the east, and the high temperature regions were found in the Western Industrial Cluster, Qianhai and Futian-Luohu Urban Municipal Center, Central Urban Cluster, and Eastern Industrial Cluster. On the seasonal scale, there was a clear LST distribution pattern with the highest surface temperatures located in the important transportation hub in all seasons. It should be noted however that LST values' range and high temperature concentration areas vary on seasonal basis. (2) The spatiotemporal distribution of UHII is generally consistent with LST, with higher SUHI intensities in spring and summer. Five profiles were drawn to analyze the distribution of the UHII in different seasons. It was found that the No. 1 path profiles that are in the western developmental axis, whose main function is to develop modern service and manufacturing industry, have higher UHII than other path profiles while the No. 5 path profiles in the southern developmental belt whose purpose was to boost finance and tourism had relatively low UHII value. (3) The LSTs from four seasons were standardized and classified for characterizing the spatiotemporal distribution of the LST levels. Among all levels of temperature zones, the high temperature zones occupy the largest proportion in spring and summer, while the medium temperature zones occupy the largest proportion in autumn and winter. (4) From the UHII spatial distribution analysis, a spatially discontinuous pattern was observed in winter and autumn, while in summer and spring, there was a compact pattern of high temperature zones. Moran's I values were higher in summer and spring, followed by winter and autumn. The center of gravity of UHII shifts converged in the Longhua, Baoan, and Nanshan Districts throughout the four seasons. Results indicated that seasonal variation could greatly affect the distribution and transfer of UHII gravity centers in Shenzhen.

Based on the findings highlighted above, it is believed that this study may provide urban planners in Shenzhen with useful information to monitor the urban thermal environment in different seasons and could serve as a reference tool in the effort to alleviate SUHI effect and improve the management of urban thermal environment in the quest to enhance the residents' well-being. Finally, given that the reasons for UHII center of gravity shifts in different seasons and the driving forces acting upon the urban thermal environment in Shenzhen cannot be exactly pinpointed in this study, further investigations are highly suggested.

\section{Conflicts of Interest}

The authors declare that there are no conflicts of interest regarding the publication of this paper.

\section{Authors' Contributions}

Yao Yuan designed the research; Yao Yuan, Chen Xi, Qian Jing, and Ndayisaba Felix processed the data, analyzed the results, and wrote the manuscript. All authors contributed to the final version of the manuscript by proofreading and bringing in constructive ideas.

\section{Acknowledgments}

This study was financially supported by Basic Research Project of Shenzhen (JCYJ201506 3011 4942312, JCYJ20150630114 942260).

\section{References}

[1] A. M. Rizwan, L. Y. C. Dennis, and C. Liu, "A review on the generation, determination and mitigation of Urban Heat Island," Journal of Environmental Sciences, vol. 20, no. 1, pp. 120128, 2008.

[2] P. Sequera, J. E. González, K. McDonald, S. Ladochy, and D. Comarazamy, "Improvements in land-use classification for estimating daytime surface temperatures and sea-breeze flows in Southern California," Earth Interactions, vol. 20, no. 16, pp. $1-32,2016$.

[3] S. Peng, S. Piao, P. Ciais et al., "Surface urban heat island across 419 global big cities," Environmental Science \& Technology, vol. 46, no. 2, pp. 696-703, 2012.

[4] Y. Ma, Y. Kuang, and N. Huang, "Coupling urbanization analyses for studying urban thermal environment and its interplay with biophysical parameters based on TM/ETM+ imagery," International Journal of Applied Earth Observation and Geoinformation, vol. 12, no. 2, pp. 110-118, 2010.

[5] Z. Qiao, G. Tian, and L. Xiao, "Diurnal and seasonal impacts of urbanization on the urban thermal environment: a case study of Beijing using MODIS data," ISPRS Journal of Photogrammetry and Remote Sensing, vol. 85, pp. 93-101, 2013.

[6] M. Xie, Y. Wang, M. Fu, and D. Zhang, "Pattern dynamics of thermal-environment effect during urbanization: a case study in Shenzhen City, China," Chinese Geographical Science, vol. 23, no. 1, pp. 1-12, 2013.

[7] K. George, L. H. Ziska, J. A. Bunce, and B. Quebedeaux, "Elevated atmospheric $\mathrm{CO}_{2}$ concentration and temperature across an urban-rural transect," Atmospheric Environment, vol. 41, no. 35, pp. 7654-7665, 2007.

[8] V. Caselles, M. J. López García, J. Meliá, and A. J. Pérez Cueva, "Analysis of the heat-island effect of the city of Valencia, Spain, through air temperature transects and NOAA satellite data," Theoretical and Applied Climatology, vol. 43, no. 4, pp. 195-203, 1991.

[9] N. B. Grimm, S. H. Faeth, N. E. Golubiewski et al., "Global change and the ecology of cities," Science, vol. 319, no. 5864, pp. 756-760, 2008.

[10] X.-L. Chen, H.-M. Zhao, P.-X. Li, and Z.-Y. Yin, "Remote sensing image-based analysis of the relationship between urban heat island and land use/cover changes," Remote Sensing of Environment, vol. 104, no. 2, pp. 133-146, 2006.

[11] M. Stathopoulou and C. Cartalis, "Daytime urban heat islands from Landsat ETM+ and Corine land cover data: an application to major cities in Greece," Solar Energy, vol. 81, no. 3, pp. 358$368,2007$.

[12] T. Oke, "The heat island of the urban boundary layer: characteristics, causes and effects," in Wind Climate in Cities, pp. 81-107, Springer, Dordrecht, Netherlands, 1995.

[13] D. M. Yow, "Urban heat islands: observations, impacts, and adaptation," Geography Compass, vol. 1, no. 6, pp. 1227-1251, 2007.

[14] N. Schwarz, S. Lautenbach, and R. Seppelt, "Exploring indicators for quantifying surface urban heat islands of European 
cities with MODIS land surface temperatures," Remote Sensing of Environment, vol. 115, no. 12, pp. 3175-3186, 2011.

[15] T. R. Oke, "The distinction between canopy and boundary-layer urban heat Islands," Atmosphere, vol. 14, no. 4, pp. 268-277, 1976.

[16] D. Ambrosini, G. Galli, B. Mancini, I. Nardi, and S. Sfarra, "Evaluating mitigation effects of urban heat islands in a historical small center with the ENVI-Met ${ }^{\circledR}$ climate model," Sustainability, vol. 6, no. 10, pp. 7013-7029, 2014.

[17] L. E. Icaza, A. Van Den Dobbelsteen, and F. Van Der Hoeven, "Integrating urban heat assessment in urban plans," Sustainability, vol. 8, no. 4, article no. 320, 2016.

[18] X. Li, W. Li, A. Middel, S. L. Harlan, A. J. Brazel, and B. L. Turner, "Remote sensing of the surface urban heat island and land architecture in Phoenix, Arizona: combined effects of land composition and configuration and cadastral-demographiceconomic factors," Remote Sensing of Environment, vol. 174, pp. 233-243, 2016.

[19] S. Alavipanah, M. Wegmann, S. Qureshi, Q. Weng, and T. Koellner, "The role of vegetation in mitigating urban land surface temperatures: a case study of Munich, Germany during the warm season," Sustainability, vol. 7, no. 4, pp. 4689-4706, 2015.

[20] D. R. Streutker, "A remote sensing study of the urban heat island of Houston, Texas," International Journal of Remote Sensing, vol. 23, no. 13, pp. 2595-2608, 2002.

[21] Q. Weng, "Thermal infrared remote sensing for urban climate and environmental studies: methods, applications, and trends," ISPRS Journal of Photogrammetry and Remote Sensing, vol. 64, no. 4, pp. 335-344, 2009.

[22] H. Shen, L. Huang, L. Zhang, P. Wu, and C. Zeng, "Long-term and fine-scale satellite monitoring of the urban heat island effect by the fusion of multi-temporal and multi-sensor remote sensed data: a 26-year case study of the city of Wuhan in China," Remote Sensing of Environment, vol. 172, pp. 109-125, 2016.

[23] L. Hu and N. A. Brunsell, "A new perspective to assess the urban heat island through remotely sensed atmospheric profiles," Remote Sensing of Environment, vol. 158, pp. 393-406, 2015.

[24] T. R. Oke, "City size and the urban heat island," Atmospheric Environment, vol. 7, no. 8, pp. 769-779, 1973.

[25] P. K. Rao, "Remote sensing of urban heat islands from an environmental satellite," Bulletin of the American Meteorological Society, vol. 53, no. 7, pp. 647-648, 1972.

[26] Q. H. Weng, D. S. Lu, and J. Schubring, "Estimation of land surface temperature-vegetation abundance relationship for urban heat island studies," Remote Sensing of Environment, vol. 89, no. 4, pp. 467-483, 2004.

[27] C. P. Lo and D. A. Quattrochi, "Land-use and land-cover change, urban heat island phenomenon, and health implications: a remote sensing approach," Photogrammetric Engineering and Remote Sensing, vol. 69, no. 9, pp. 1053-1063, 2003.

[28] H. Wu, L.-P. Ye, W.-Z. Shi, and K. C. Clarke, "Assessing the effects of land use spatial structure on urban heat islands using $\mathrm{HJ}-1 \mathrm{~B}$ remote sensing imagery in Wuhan, China," International Journal of Applied Earth Observation and Geoinformation, vol. 32, pp. 67-78, 2014.

[29] K. Zakšek and K. Oštir, "Downscaling land surface temperature for urban heat island diurnal cycle analysis," Remote Sensing of Environment, vol. 117, pp. 114-124, 2012.

[30] J. Zhou, Y. Chen, X. Zhang, and W. Zhan, "Modelling the diurnal variations of urban heat islands with multi-source satellite data," International Journal of Remote Sensing, vol. 34, no. 21, pp. 7568-7588, 2013.
[31] T. W. Owen, T. N. Carlson, and R. R. Gillies, "An assessment of satellite remotely-sensed land cover parameters in quantitatively describing the climatic effect of urbanization," International Journal of Remote Sensing, vol. 19, no. 9, pp. 16631681, 1998.

[32] C. J. Tomlinson, L. Chapman, J. E. Thornes, and C. J. Baker, "Derivation of Birmingham's summer surface urban heat island from MODIS satellite images," International Journal of Climatology, vol. 32, no. 2, pp. 214-224, 2012.

[33] S. Cheval and A. Dumitrescu, "The July urban heat island of Bucharest as derived from modis images," Theoretical and Applied Climatology, vol. 96, no. 1-2, pp. 145-153, 2009.

[34] L. Liu and Y. Z. Zhang, "Urban heat island analysis using the landsat TM data and ASTER data: a case study in Hong Kong," Remote Sensing, vol. 3, no. 7, pp. 1535-1552, 2011.

[35] Q. Weng, "A remote sensing? GIS evaluation of urban expansion and its impact on surface temperature in the Zhujiang Delta, China," International Journal of Remote Sensing, vol. 22, no. 10, pp. 1999-2014, 2001.

[36] D. P. Roy, M. A. Wulder, T. R. Loveland et al., "Landsat8: science and product vision for terrestrial global change research," Remote Sensing of Environment, vol. 145, pp. 154-172, 2014.

[37] X. Ouyang, L. Jia, Y. Pan, and G. Hu, "Retrieval of land surface temperature over the Heihe River basin using HJ-1B thermal infrared data," Remote Sensing, vol. 7, no. 1, pp. 300-318, 2015.

[38] Z. Liang, Y. Lin, Y. Ma et al., "The association between ambient temperature and preterm birth in Shenzhen, China: a distributed lag non-linear time series analysis," Environmental Health: A Global Access Science Source, vol. 15, no. 1, article 84, 2016.

[39] Y. Ban and A. Jacob, "Object-based fusion of multitemporal multiangle ENVISAT ASAR and HJ-1B multispectral data for urban land-cover mapping," IEEE Transactions on Geoscience and Remote Sensing, vol. 51, no. 4, pp. 1998-2006, 2013.

[40] L. Lin, Y. Meng, A. Yue et al., "A spatio-temporal model for forest fire detection using HJ-IRS satellite data," Remote Sensing, vol. 8, no. 5, article 403, 2016.

[41] S. Yu, X. Xin, and Q. Liu, "Estimation of clear-sky longwave downward radiation from HJ-1B thermal data," Science China Earth Sciences, vol. 56, no. 5, pp. 829-842, 2013.

[42] Four seasons is how to divide, http://www.szmb.gov.cn/article/ ZaiXianBanShi/YeWuZhiShiKu/YuJingYuBaoLei/2015/11/17/ 564a88573d722.html.

[43] B.-L. Zhong, "Division of seasons at shenzhen based on 5 days running mean temperature," Meteorological Monthly, vol. 21, pp. 22-23, 1995.

[44] X. Chen, S. Bao, H. Li et al., "LUCC impact on sediment loads in subtropical rainy areas," Photogrammetric Engineering and Remote Sensing, vol. 73, no. 3, pp. 319-327, 2007.

[45] C. O. Justice, E. Vermote, J. R. G. Townshend et al., “The moderate resolution imaging spectroradiometer (MODIS): land remote sensing for global change research," IEEE Transactions on Geoscience and Remote Sensing, vol. 36, no. 4, pp. 1228-1249, 1998.

[46] J. Tan, Y. Zheng, X. Tang et al., "The urban heat island and its impact on heat waves and human health in Shanghai," International Journal of Biometerology, vol. 54, no. 1, pp. 75-84, 2010.

[47] Z. Qin, A. Karnieli, and P. Berliner, "A mono-window algorithm for retrieving land surface temperature from Landsat TM 
data and its application to the Israel-Egypt border region," International Journal of Remote Sensing, vol. 22, no. 18, pp. 37193746, 2001.

[48] J. A. Sobrino, Z.-L. Li, M. P. Stoll, and F. Becker, "Multi-channel and multi-angle algorithms for estimating sea and land surface temperature with atsr data," International Journal of Remote Sensing, vol. 17, no. 11, pp. 2089-2114, 1996.

[49] A. Gillespie, S. Rokugawa, T. Matsunaga, J. S. Cothern, S. Hook, and A. B. Kahle, "A temperature and emissivity separation algorithm for advanced spaceborne thermal emission and reflection radiometer (ASTER) images," IEEE Transactions on Geoscience and Remote Sensing, vol. 36, no. 4, pp. 1113-1126, 1998.

[50] J. C. Jiménez-Muñoz and J. A. Sobrino, "A generalized singlechannel method for retrieving land surface temperature from remote sensing data," Journal of Geophysical Research: Atmospheres, vol. 108, p. D22, 2003.

[51] Y. Lu, X. Feng, P. Xiao, C. Shen, and J. Sun, "Urban heat island in summer of Nanjing based on TM data," in Proceedings of 2009 Joint Urban Remote Sensing Event, pp. 1-15, May 2009.

[52] J. A. Sobrino, J. C. Jiménez-Muñoz, and L. Paolini, "Land surface temperature retrieval from LANDSAT TM 5," Remote Sensing of Environment, vol. 90, no. 4, pp. 434-440, 2004.

[53] J. Zhou, W. Zhan, D. Hu, and X. Zhao, "Improvement of monowindow algorithm for retrieving land surface temperature from HJ-1B satellite data," Chinese Geographical Science, vol. 20, no. 2, pp. 123-131, 2010.

[54] H. Rhinane, A. Hilali, H. Bahi, and A. Berrada, "Contribution of landsat TM data for the detection of urban heat islands areas case of Casablanca," Journal of Geographic Information System, vol. 04, no. 01, pp. 20-26, 2012.

[55] Q. Q. Sun, J. J. Tan, and Y. H. Xu, "An ERDAS image processing method for retrieving LST and describing urban heat evolution: a case study in the Pearl River Delta Region in South China," Environmental Earth Sciences, vol. 59, no. 5, pp. 1047-1055, 2009.

[56] J. A. Sobrino, N. Raissouni, and Z.-L. Li, "A comparative study of land surface emissivity retrieval from NOAA data," Remote Sensing of Environment, vol. 75, no. 2, pp. 256-266, 2001.

[57] Z. Xu, H. Zhao, and N. Li, "Study of inversing land surface emissivity from ASTER data," Infrared and Laser Engineering, vol. 35, pp. 517-522, 2006.

[58] Z. Qin, W. Li, Z. Chen, and H. Tang, "Land surface emissivity estimation for LST retrieval from Landsat TM6 data," Remote Sensing for Land and Resources, vol. 3, pp. 28-32, 2004.

[59] S.-B. Duan, G.-J. Yan, Y.-G. Qian, Z.-L. Li, X.-G. Jiang, and X.-W. $\mathrm{Li}$, "Two single - channel algorithms for retrieving land surface temperature using HJ - 1B data," Progress in Natural Science, vol. 18, pp. 1001-1008, 2008.

[60] U. Rajasekar and Q. Weng, "Urban heat island monitoring and analysis using a non-parametric model: a case study of Indianapolis," ISPRS Journal of Photogrammetry and Remote Sensing, vol. 64, no. 1, pp. 86-96, 2009.

[61] M. Lazzarini, P. R. Marpu, and H. Ghedira, “Temperatureland cover interactions: the inversion of urban heat island phenomenon in desert city areas," Remote Sensing of Environment, vol. 130, pp. 136-152, 2013.

[62] W. Wang, S. Liang, and T. Meyers, "Validating MODIS land surface temperature products using long-term nighttime ground measurements," Remote Sensing of Environment, vol. 112, no. 3, pp. 623-635, 2008.
[63] J. Yang, P. Gong, J. Zhou, H. Huang, and L. Wang, "Detection of the urban heat island in Beijing using HJ-1B satellite imagery," Science China Earth Sciences, vol. 53, no. 1, pp. 67-73, 2010.

[64] F. Chen, S. Yang, Z. Su, and B. He, "A new single-channel method for estimating land surface temperature based on the image inherent information: the HJ-1B case," ISPRS Journal of Photogrammetry and Remote Sensing, vol. 101, pp. 80-88, 2015.

[65] D. D. Alexakis and I. K. Tsanis, "Comparison of multiple linear regression and artificial neural network models for downscaling TRMM precipitation products using MODIS data," Environmental Earth Sciences, vol. 75, no. 14, article no. 1077, pp. 1-13, 2016.

[66] T. Bao, X. Li, J. Zhang, Y. Zhang, and S. Tian, "Assessing the distribution of urban green spaces and its anisotropic cooling distance on urban heat island pattern in Baotou, China," ISPRS International Journal of Geo-Information, vol. 5, no. 2, article no. 12, 2016.

[67] M. L. Imhoff, P. Zhang, R. E. Wolfe, and L. Bounoua, "Remote sensing of the urban heat island effect across biomes in the continental USA," Remote Sensing of Environment, vol. 114, no. 3, pp. 504-513, 2010.

[68] P. Zhang, M. L. Imhoff, R. E. Wolfe, and L. Bounoua, "Characterizing urban heat islands of global settlements using MODIS and nighttime lights products," Canadian Journal of Remote Sensing, vol. 36, no. 3, pp. 185-196, 2010.

[69] Rural disappeared, Shenzhen became the first no rural city in China, http://news.sohu.com/20100906/n274737376.shtml.

[70] R. Amiri, Q. Weng, A. Alimohammadi, and S. K. Alavipanah, "Spatial-temporal dynamics of land surface temperature in relation to fractional vegetation cover and land use/cover in the Tabriz urban area, Iran," Remote Sensing of Environment, vol. 113, no. 12, pp. 2606-2617, 2009.

[71] A. M. Coutts, R. J. Harris, T. Phan, S. J. Livesley, N. S. G. Williams, and N. J. Tapper, "Thermal infrared remote sensing of urban heat: hotspots, vegetation, and an assessment of techniques for use in urban planning," Remote Sensing of Environment, vol. 186, pp. 637-651, 2016.

[72] C. Shen, C. Li, and Y. Si, "Spatio-temporal autocorrelation measures for nonstationary series: a new temporally detrended spatio-temporal Moran's index," Physics Letters A, vol. 380, no. 1-2, pp. 106-116, 2016.

[73] Z. Qiao, G. Tian, L. Zhang, and X. Xu, "Influences of urban expansion on urban heat island in Beijing during 1989-2010," Advances in Meteorology, vol. 2014, Article ID 187169, p. 11, 2014.

[74] A. Ayanlade, "Variation in diurnal and seasonal urban land surface temperature: landuse change impacts assessment over Lagos metropolitan city," Modeling Earth Systems and Environment, vol. 2, no. 4, 2016.

[75] M. Xie, Y. Wang, Q. Chang, M. Fu, and M. Ye, "Assessment of landscape patterns affecting land surface temperature in different biophysical gradients in Shenzhen, China," Urban Ecosystems, vol. 16, no. 4, pp. 871-886, 2013.

[76] X. Lu, K.-C. Chow, T. Yao, J. C. H. Fung, and A. K. H. Lau, "Seasonal variation of the land-sea breeze circulation in the Pearl River Delta region," Journal of Geophysical Research: Atmospheres, vol. 114, no. 17, Article ID D17112, 2009.

[77] Meteorological Bureau of Shenzhen Municipality, http://www .szmb.gov.cn/en/.

[78] D. Zhou, L. Zhang, D. Li, D. Huang, and C. Zhu, "Climatevegetation control on the diurnal and seasonal variations of surface urban heat islands in China," Environmental Research Letters, vol. 11, no. 7, Article ID 074009, 2016. 
[79] D. Zhou, S. Zhao, S. Liu, L. Zhang, and C. Zhu, "Surface urban heat island in China's 32 major cities: spatial patterns and drivers," Remote Sensing of Environment, vol. 152, pp. 51-61, 2014.

[80] Z. Chen, C. Gong, J. Wu, and S. Yu, "The influence of socioeconomic and topographic factors on nocturnal urban heat islands: a case study in Shenzhen, China," International Journal of Remote Sensing, vol. 33, no. 12, pp. 3834-3849, 2012.

[81] J. Li, C. Song, L. Cao, F. Zhu, X. Meng, and J. Wu, "Impacts of landscape structure on surface urban heat islands: a case study of Shanghai, China," Remote Sensing of Environment, vol. 115, no. 12, pp. 3249-3263, 2011.

[82] F. Yuan and M. E. Bauer, "Comparison of impervious surface area and normalized difference vegetation index as indicators of surface urban heat island effects in Landsat imagery," Remote Sensing of Environment, vol. 106, no. 3, pp. 375-386, 2007.

[83] A. J. Arnfield, "Two decades of urban climate research: a review of turbulence, exchanges of energy and water, and the urban heat island," International Journal of Climatology, vol. 23, no. 1, pp. 1-26, 2003.

[84] J. M. Shepherd, "A review of current investigations of urbaninduced rainfall and recommendations for the future," Earth Interactions, vol. 9, no. 12, pp. 1-27, 2005.

[85] I. D. Stewart and T. R. Oke, "Local climate zones for urban temperature studies," Bulletin of the American Meteorological Society, vol. 93, no. 12, pp. 1879-1900, 2012.

[86] P. Grady Dixon and T. L. Mote, "Patterns and causes of Atlanta's urban heat island-initiated precipitation," Journal of Applied Meteorology and Climatology, vol. 42, no. 9, pp. 1273-1284, 2003.

[87] H. Tran, D. Uchihama, S. Ochi, and Y. Yasuoka, "Assessment with satellite data of the urban heat island effects in Asian mega cities," International Journal of Applied Earth Observation and Geoinformation, vol. 8, no. 1, pp. 34-48, 2006.

[88] S. Amaral, G. Câmara, A. M. V. Monteiro, J. A. Quintanilha, and C. D. Elvidge, "Estimating population and energy consumption in Brazilian Amazonia using DMSP night-time satellite data," Computers, Environment and Urban Systems, vol. 29, no. 2, pp. 179-195, 2005.

[89] J. E. Nichol, "High-resolution surface temperature patterns related to urban morphology in a tropical city: a satellite-based study," Journal of Applied Meteorology and Climatology, vol. 35, no. 1, pp. 135-146, 1996.

[90] Y. Kikegawa, Y. Genchi, H. Yoshikado, and H. Kondo, "Development of a numerical simulation system toward comprehensive assessments of urban warming countermeasures including their impacts upon the urban buildings' energy-demands," Applied Energy, vol. 76, no. 4, pp. 449-466, 2003.

[91] P. A. Mirzaei and F. Haghighat, "Approaches to study Urban Heat Island - abilities and limitations," Building and Environment, vol. 45, no. 10, pp. 2192-2201, 2010.

[92] H. Akbari, L. S. Rose, and H. Taha, "Analyzing the land cover of an urban environment using high-resolution orthophotos," Landscape and Urban Planning, vol. 63, no. 1, pp. 1-14, 2003.

[93] A. Synnefa, A. Dandou, M. Santamouris, M. Tombrou, and N. Soulakellis, "On the use of cool materials as a heat island mitigation strategy," Journal of Applied Meteorology and Climatology, vol. 47, no. 11, pp. 2846-2856, 2008.

[94] H. Akbari, S. Menon, and A. Rosenfeld, "Global cooling: increasing world-wide urban albedos to offset $\mathrm{CO}_{2}$," Climatic Change, vol. 94, no. 3-4, pp. 275-286, 2009.

[95] H. Akbari and S. Konopacki, "Calculating energy-saving potentials of heat-island reduction strategies," Energy Policy, vol. 33, no. 6, pp. 721-756, 2005.
[96] M. Zinzi and S. Agnoli, "Cool and green roofs. An energy and comfort comparison between passive cooling and mitigation urban heat island techniques for residential buildings in the Mediterranean region," Energy and Buildings, vol. 55, pp. 66-76, 2012.

[97] C. Yu and W. N. Hien, "Thermal benefits of city parks," Energy and Buildings, vol. 38, no. 2, pp. 105-120, 2006.

[98] Y. Ashie, V. T. Ca, and T. Asaeda, "Building canopy model for the analysis of urban climate," Journal of Wind Engineering \& Industrial Aerodynamics, vol. 81, pp. 237-248, 1999.

[99] H. Tong, A. Walton, J. Sang, and J. C. L. Chan, "Numerical simulation of the urban boundary layer over the complex terrain of Hong Kong," Atmospheric Environment, vol. 39, no. 19, pp. 3549-3563, 2005. 

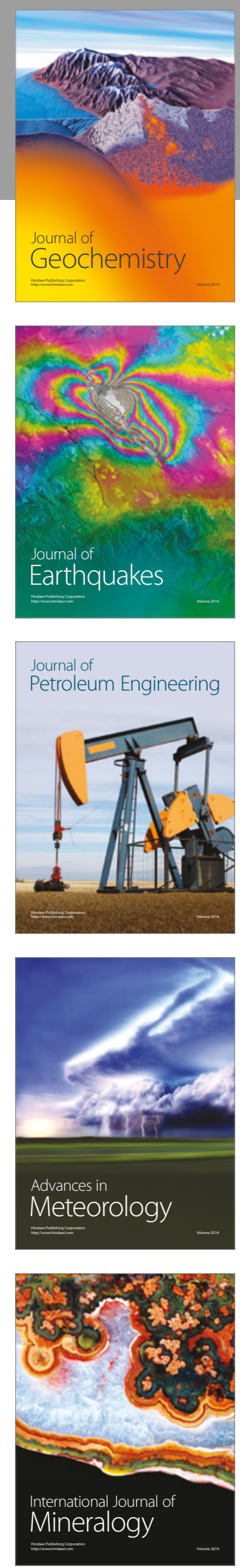
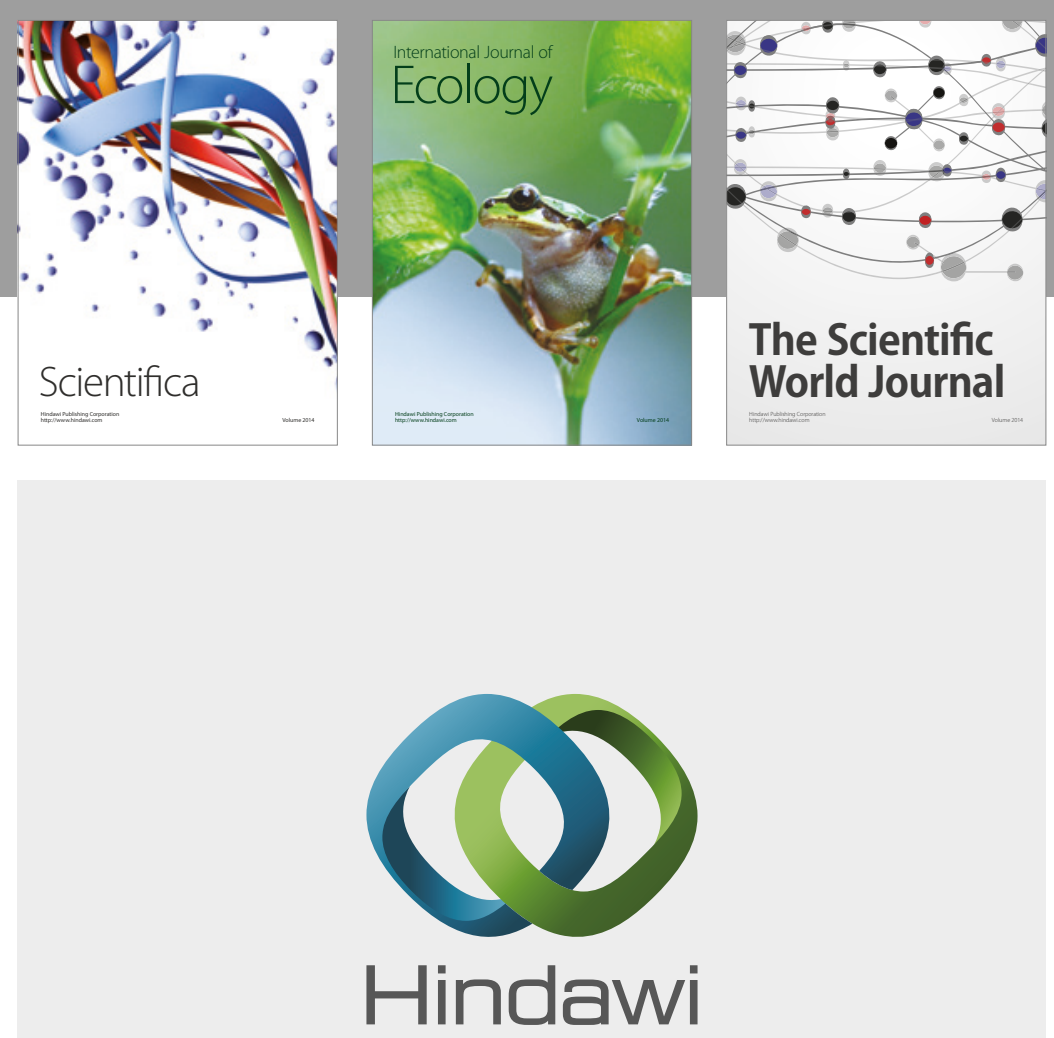

Submit your manuscripts at

https://www.hindawi.com
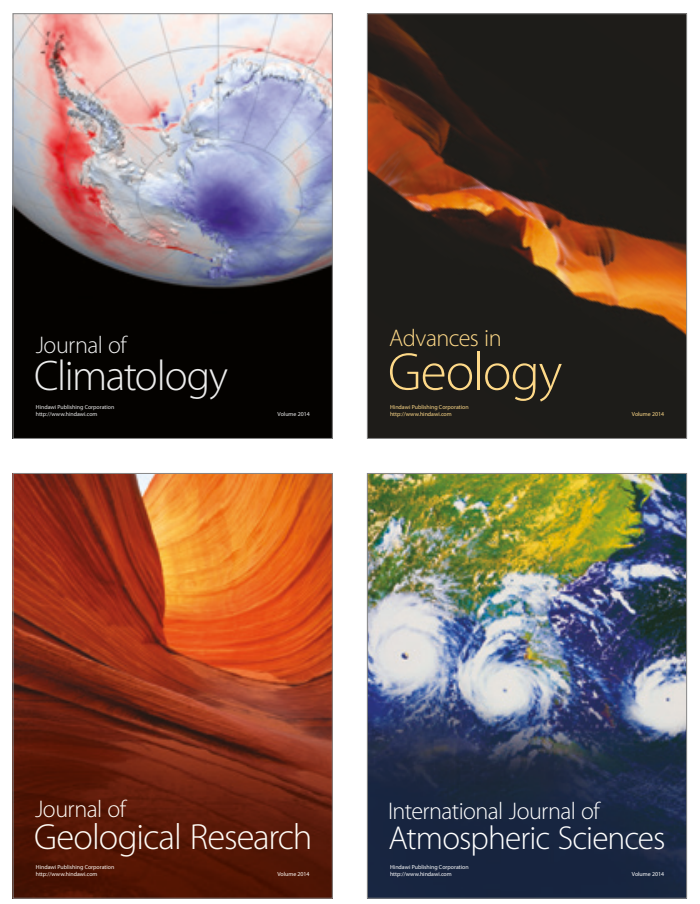

The Scientific

World Journal
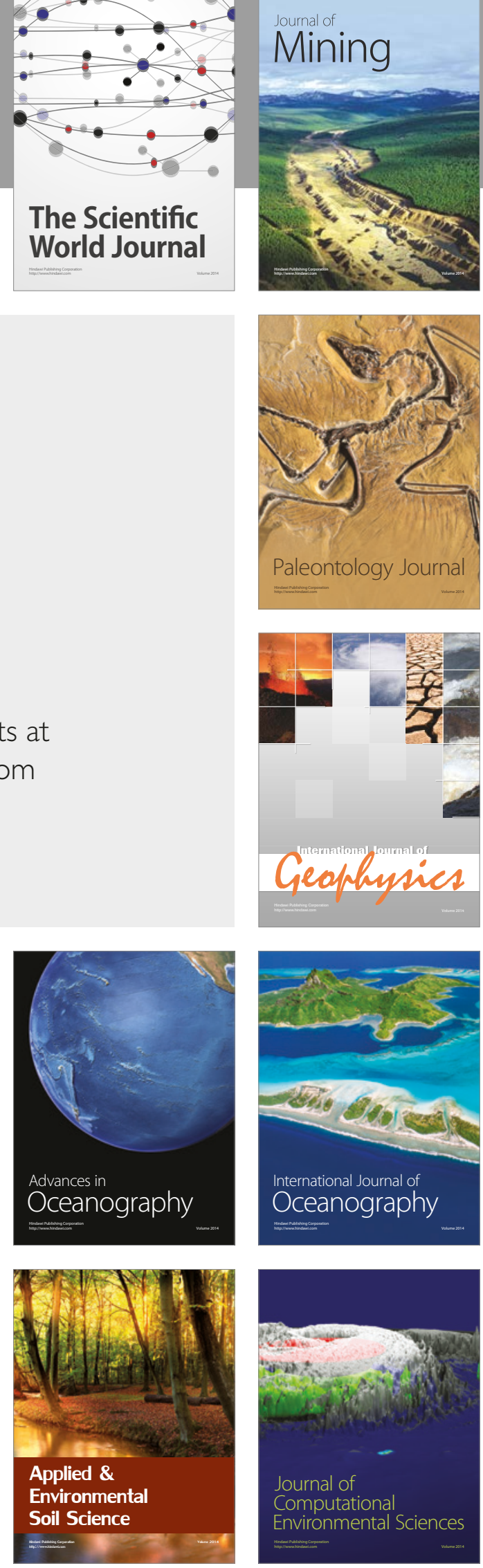